\title{
Larger brain size indirectly increases vulnerability to extinction in mammals
}

Article

Accepted Version

Gonzalez-Voyer, A., Gonzalez-Suarez, M., Vilá, C. and Revilla, E. (2016) Larger brain size indirectly increases vulnerability to extinction in mammals. Evolution. ISSN 0014-3820 doi:

https://doi.org/10.1111/evo.12943 Available at https://centaur.reading.ac.uk/65634/

It is advisable to refer to the publisher's version if you intend to cite from the work. See Guidance on citing.

To link to this article DOI: http://dx.doi.org/10.1111/evo.12943

Publisher: Wiley

All outputs in CentAUR are protected by Intellectual Property Rights law, including copyright law. Copyright and IPR is retained by the creators or other copyright holders. Terms and conditions for use of this material are defined in the End User Agreement.

\section{www.reading.ac.uk/centaur}

\section{CentAUR}

Central Archive at the University of Reading

Reading's research outputs online 


\section{Larger brain size indirectly increases vulnerability to extinction in mammals.}

Alejandro Gonzalez-Voyer ${ }^{1,2,3 \dagger}$, Manuela González-Suárez ${ }^{4,5 \dagger}$, Carles Vilà ${ }^{1}$ and Eloy Revilla ${ }^{4}$.

Affiliations: ${ }^{1}$ Conservation and Evolutionary Genetics Group, Department of Integrative Ecology,

Estación Biológica de Doñana (EBD-CSIC), c/Américo Vespucio s/n, 41092, Sevilla, Spain.

${ }^{2}$ Department of Zoology / Ethology, Stockholm University, Svante Arrheniusväg 18 B, SE-10691, Stockholm, Sweden.

${ }^{3}$ Laboratorio de Conducta Animal, Instituto de Ecología, Circuito Exterior S/N, Universidad Nacional Autónoma de México, México, D. F., 04510, México.

${ }^{4}$ Department of Conservation Biology, Estación Biológica de Doñana (EBD-CSIC), c/Américo

Vespucio s/n, 41092, Sevilla, Spain.

${ }^{5}$ Ecology and Evolutionary Biology, School of Biological Sciences, University of Reading, Whiteknights, Reading RG6 6AS, UK

Email addresses: AG-V alejandro.gonzalez@iecologia.unam.mx , MG-S

manuela.gonzalez@reading.ac.uk,CV carles.vila@ebd.csic.es, ER revilla@ebd.csic.es

Corresponding authors: Alejandro Gonzalez-Voyer, Laboratorio de Conducta Animal, Instituto de

Ecología, Circuito Exterior SN, Universidad Nacional Autónoma de México, México, D. F., 04510, Mexico. Tel: +52 55 56229044, E-mail: alejandro.gonzalez@iecologia.unam.mx

Manuela González-Suárez, Ecology and Evolutionary Biology, School of

Biological Sciences, University of Reading, Whiteknights, Reading RG6 6AS, UK. Tel: +44 (0) 118

378 6437, E-mail: manuela.gonzalez@ reading.ac.uk.

†These authors contributed equally.

Running title: Brain size and vulnerability to extinction.

Number of words in Text: 6602 


\begin{abstract}
Although previous studies have addressed the question of why large brains evolved, we have limited understanding of potential beneficial or detrimental effects of enlarged brain size in the face of current threats. Using novel phylogenetic path analysis, we evaluated how brain size directly and indirectly, via its effects on life-history and ecology, influences vulnerability to extinction across 474 mammalian species. We found that larger brains, controlling for body size, indirectly increase vulnerability to extinction by extending the gestation period, increasing weaning age, and limiting litter sizes. However, we found no evidence of direct, beneficial or detrimental, effects of brain size on vulnerability to extinction, even when we explicitly considered the different types of threats that lead to vulnerability. Order-specific analyses revealed qualitatively similar patterns for Carnivora and Artiodactyla. Interestingly, for Primates, we found that larger brain size was directly (and indirectly) associated with increased vulnerability to extinction. Our results indicate that under current conditions the constraints on life-history imposed by large brains outweigh the potential benefits, undermining the resilience of the studied mammals. Contrary to the selective forces that have favoured increased brain size throughout evolutionary history, at present, larger brains have become a burden for mammals.
\end{abstract}

Keywords: body size allometry, extinction risk, IUCN Red List, life-history traits, phylogenetic path analysis. 


\section{Introduction}

Humans stand out among other animals because of their large brain size in relation to body mass. Yet, variation in brain size is extensive across the animal kingdom (Striedter 2005). Interspecific differences in brain size for a given body size (henceforth simply referred to as brain size), have long puzzled evolutionary biologists. Current explanations suggest that large brains have evolved through a balance between selection and constraints. Larger brains are associated with greater behavioural flexibility which is proposed to act as a buffer against environmental challenges (Sol 2009). Species with relatively larger brains also show higher frequency of innovative behaviour and tool use (Reader and Laland 2002; Lefebvre et al. 2004). As a result larger brains are predicted to be associated with improved survival and higher ability to persist in novel environments. Both predictions have been confirmed in diverse taxa in which larger brain sizes are positively associated with longevity (González-Lagos et al. 2010; but see Barton and Capellini 2011), lower likelihood of population declines (Shultz et al. 2005; Pocock 2011), higher ability to colonize novel environments (Maklakov et al. 2011), higher establishment success for invasive and introduced species (Sol et al. 2002; Sol et al. 2008; Amiel et al. 2011), and lower mortality rates (Sol et al. 2007).

Although large brains can confer advantages, there are also important energetic constraints that can limit the increase in brain size in natural populations. Along with the digestive tract, the brain is the most energetically costly organ in the body. In adult humans for example, the brain represents about $2 \%$ of total body mass but consumes nearly $20 \%$ of the total energy intake (Aiello and Wheeler 1995). In mammals, the increased energetic costs of growing and maintaining larger brains have been found to be associated with increased maternal investment in the form of longer gestation and lactation periods beyond allometric effects (Barton and Capellini 2011). Larger brains have also been associated with reduced population growth rates (Isler and van Schaik 2009a), although brain size and body size were simultaneously included as predictors in the models but showed inverse relationships with population 
growth rate. As far as we know, the only available experimental evidence of the costs of enlarged brain size comes from a selection experiment with guppies which found that large-brain lines present a $19 \%$ decrease in offspring number compared with small-brain lines (Kotrschal et al. 2013). Despite these constraints, mammalian brain size has increased over evolutionary time as species diversified to fill a variety of ecological niches (Montgomery et al. 2010; Rowe et al. 2011). However, at present, anthropogenic activities are causing rapid changes in natural environments that have resulted in accelerated extinction rates (Barnosky et al. 2011; Urban 2015). Under these conditions, the balance between the benefits (e.g., plasticity) and energetic constraints that allowed the evolution of large brains may have been altered. Very little is known about the current net outcome of positive selection for larger brain size and constraints imposed by energetic costs, which is worrying in the face of rapid environmental changes.

The current rate of biodiversity loss has placed a premium on understanding what determines species vulnerability. When it comes to extinction, species are not all equally at risk (Purvis et al. 2000a). Vulnerability to extinction is largely determined by a potentially complex combination of intrinsic species traits (e.g. life-history characteristics), extrinsic factors (such as anthropogenic impacts on a species' habitat), and their interactions (Foufopoulos and Ives 1999; Owens and Bennett 2000; Cardillo et al. 2006; Davidson et al. 2009; Murray et al. 2011; González-Suárez et al. 2013; GonzálezSuárez and Revilla 2013; González-Suárez and Revilla 2014). Previous studies analysing the correlates of vulnerability to extinction have investigated the association with life-history and ecological traits, often emphasizing the role of allometric effects and body size differences (Davidson et al. 2009; Fritz et al. 2009). Large body size has been linked to greater risk of extinction in birds and mammals (Bennett and Owens 1997; Boyer 2010; González-Suárez and Revilla 2013) because larger species often live at lower average population densities, are disproportionately exploited by humans, and have slower intrinsic population growth rates with smaller litter (or clutch) sizes, longer gestation 
(incubation) times and later ages at first reproduction (Bodmer et al. 1997; Bielby et al. 2007;

González-Suárez et al. 2013). All these factors are in turn associated with higher risk of extinction (Cardillo et al. 2005; Davidson et al. 2009; Fritz et al. 2009; González-Suárez and Revilla 2013). Comparatively, the aforementioned studies paid little attention to brain size, even though previous works, such as those mentioned earlier, analysing the evolution of brain size revealed the seemingly distinct roles of body size and brain size in shaping life-history and possibly population dynamics. These divergent lines of research must be reconciled to adequately assess current risk of extinction and explicitly evaluate the potential role of brain size under current conditions.

A species' vulnerability to extinction, as defined by the International Union for Conservation of Nature (IUCN) global Red List status (IUCN 2010), is associated with its life-history and ecology (Fig. 1A). As discussed above both brain and body size can influence vulnerability indirectly via their effect on life-history and ecological traits. In addition, body size may also directly influence vulnerability, for example because of increased harvest risk for larger species (González-Suárez et al. 2013), while brain size might directly influence vulnerability through the beneficial effects of behavioural plasticity (Shultz et al. 2005; Pocock 2011). Addressing the role of brain size on current vulnerability to extinction necessarily requires simultaneously analysing potential benefits, as well as allometric and energetic constraints acting on brain size, while also disentangling direct from indirect relationships among traits, including body size. Previous attempts to explore this question may have been impeded by methodological limitations, which have been recently removed by developments in comparative methods including new tools for phylogenetically-explicit path analyses (von Hardenberg and Gonzalez-Voyer 2013; Gonzalez-Voyer and von Hardenberg 2014). Phylogenetic path analysis allows a holistic approach, unravelling direct and indirect effects while explicitly accounting for the nonindependence of species data due to shared ancestry. Our study takes advantage of this approach to ask whether brain size influences current vulnerability to extinction beyond allometric effects. If the 
benefits of behavioural innovation and plasticity outweigh the energetic costs of growing and maintaining a large brain, then species with relatively large brains could exhibit higher resilience to current anthropogenic changes and threats. On the other hand, if the costs are higher, large brained mammals could be more vulnerable to extinction.

To address this question we defined and compared three alternative scenarios (Fig. 1A) that propose different ways in which brain and body size could influence life-history and ecological traits, and thus, indirectly influence vulnerability to extinction: 1) Body mass allometry: this scenario reflects the current paradigm in macroecological comparative studies of extinction risk that proposes that body size is directly related to life-history and ecological traits. 2) Brain costs and benefits: this alternative scenario proposes that brain size is directly related to life-history and ecology, with body size having only indirect effects. 3) Brain and allometry: this intermediate scenario proposes that both brain and body mass influence life-history and ecological traits. Within each scenario we also tested for possible direct associations between brain, body mass, or both traits, and vulnerability, because direct associations may occur independently of any influences of brain and body size on life-history and ecology. Our results show support for the combined influence of brain and body mass on life-history and ecological traits, showing that today many mammals with large brains -after accounting for body size- have increased vulnerability to extinction because currently the costs of large brains outweigh their potential benefits.

\section{Material and methods}

Vulnerability to extinction is a function of a species' total population size and potential population growth rate. Other criteria used to characterize the level of threat, such as size and fragmentation of the distribution range are closely associated with total population size. Unfortunately, direct estimates of total population size and population growth rate are not available for most species. Therefore, we had 
to use proxies for which sufficient data were available. We used population density as a proxy for total abundance (because given a fixed area species with higher densities are more abundant). To represent potential population growth rate we used the species' position along the slow-fast life-history continuum. In mammals two general axes characterize the slow-fast continuum: reproductive timing and reproductive output, each of which may in turn be represented by diverse life-history traits (Bielby et al. 2007). In particular, the timing of the reproductive bouts is generally best represented by weaning age and interbirth interval, and secondarily by age of sexual maturity; while the trade-off between offspring size and offspring number (reproductive output) is represented by neonate body mass, gestation length and litter size. Unfortunately, data for all of these traits are not available for all species and this limitation could influence our analysis (González-Suárez et al. 2012). Therefore, to select the most appropriate, and most widely available, candidate traits to represent a species' position along the life-history continuum, we conducted a literature review in the fall of 2013 . We searched for studies that explore how traits associate with vulnerability to extinction at a global scale in mammals. From the 10 studies (Purvis et al. 2000b; Jones et al. 2003; Cardillo et al. 2004; Cardillo et al. 2005; Cardillo et al. 2006; Cardillo et al. 2008; Davidson et al. 2009; Fritz et al. 2009; Davidson et al. 2012; GonzálezSuárez and Revilla 2013) that met our criteria we gathered information on which traits were tested and found to be significantly associated with vulnerability (for details see Supplementary Methods and Table S1 in Supporting Information). We selected traits most often found to be associated with vulnerability to extinction and for which data were available for most species (if two relevant traits were similarly supported we chose the trait with more available data). Using these criteria we characterized reproductive timing by weaning age, and reproductive output by gestation length and litter size (Table S1). A recent review of the comparative literature of extinction risk in mammals (Verde Arregoitia 2016), which analysed 68 studies (including taxa- and region-specific analyses we did not consider in our review), supports our selection criteria showing the traits we analysed here are consistently identified as relevant in explaining extinction risk in mammals. 
Species' trait information were collected from published data, such as the PanTHERIA database (Jones et al. 2009) with an additional literature search to fill data gaps. The raw data files reflecting all records located for each species and their source, as well as the species-level dataset used in our analyses are available as Supplementary Data. Species-level values were calculated as the arithmetic mean (median for population density because of its very skewed distribution) of all available measures of central tendency (mean, median, mode or mid-range) for each species describing: adult brain mass (measured in grams), adult body mass (measured in grams), population density (defined as the number of individuals per $\mathrm{km}^{2}$ ), weaning age (age at which individuals stop nursing and start to feed independently, measured in days), gestation length (length of time of active foetal growth, in days), and litter size (number of offspring born per litter per female). All species-level values were log transformed to better adjust to the assumptions of the evolutionary model (Brownian motion). Although sexual size dimorphism could influence our estimates of brain and body size, separate estimates of brain size for adult males and females are available for very few species. Nonetheless, our interest here lies in analysing the balance between benefits and costs of enlarged brain size at a species level, as vulnerability to extinction is a species characteristic and for most species differences in size between the sexes are small compared to the differences across mammalian species (see below). In total we gathered brain size data for 596 species, but data on all considered traits (including phylogenetic relationships) were available for 474 species. These 474 species are a diverse group spanning six orders of magnitude in body mass (eight orders of magnitude for population density) and including members of 21 extant orders of mammals (Fig. S1). Nevertheless, certain taxonomic groups such as Artiodactyla, Carnivora or Primates were overrepresented while Rodents and Chiroptera were underrepresented. This bias in data availability is not unique to our database but has been previously described for the PanTHERIA database (González-Suárez et al. 2012) and likely reflects biases in research topics. To define vulnerability to extinction we used the IUCN Red List which assigns extant species to different 
status categories (IUCN 2010). We included species classified as least concern (Status $=0$ ), near threatened (Status $=1)$, vulnerable (Status $=2)$, endangered (Status $=3$ ), and critically endangered (Status $=4$ ) in our analyses. Species with available data for the traits considered here but classified as 'Data Deficient' $(N=7)$ were excluded. Our dataset did not include any species classified as 'Extinct' and 'Extinct in the Wild' by the IUCN.

Red List status is an ordinal categorization of an underlying continuous variable, extinction risk, which is unfortunately unknown for most species. The phylogenetic generalized least squares methods we employed to test the conditional independencies of the path models require continuous response variables (Martins and Hansen 1997), thus we assumed that Red List status (coded as indicated above) actually reflects continuous variation in vulnerability to extinction (see also Purvis et al. 2000b; Fritz et al. 2009). At present, phylogenetically-corrected models with ordinal response variables can only be analysed in a Bayesian framework, however the path analysis method we employ requires frequentist tests of the conditional independencies (Shipley 2000; von Hardenberg and Gonzalez-Voyer 2013). Following the phylogenetic path analyses we evaluated the support of any identified relationship between species' traits and Red List status using phylogenetically-corrected Bayesian ordinal regression models implemented in the procedure MCMCglmm (Hadfield 2010) in R (R Development Core Team 2011). We modelled Red List status as an ordinal response (family=“ordinal”) and included population density, length of gestation, weaning age, body and brain mass as independent variables. The phylogeny was included as a random factor via the pedigree command. For the priors we fixed the residual variance to 1 (as suggested by $\mathrm{J}$. Hadfield; R-component V=1, fix=1), the random effect variance was set to 10 with a low credibility (i.e. a non-informative prior; G-component $\mathrm{V}=10$, $\mathrm{nu}=0.02$ ). Changes in the values of the priors had little effect on the posterior estimates. Because we assume that Red List status reflects continuous variation in vulnerability to extinction, the modeled categories represent a latent continuous variable, and a linear regression is fit between the latent 
continuous variable (with a probit link modification) and the independent variables. We ran the chain for 100000 iterations, thinning of 200 and burnin of 20000. Convergence was verified visually by plotting parameters using the package coda (Plummer et al. 2006). Effective sample sizes for all parameters were $>250$. In the Results we present the best estimates of the regression coefficients $(\beta)$ with $95 \%$ credible intervals (95\% CI). Standard interpretation of an ordinal regression coefficient is that for a one unit increase in the predictor, the response variable level is expected to change by its respective regression coefficient in the ordered probit scale while the other variables in the model are held constant. In all cases evolutionary relationships were based on an updated mammalian phylogenetic supertree (Bininda-Emonds et al. 2007; Fritz et al. 2009).

Species are listed within the IUCN Red List status categories based on five different criteria (AE) of which criteria C and D are relative to population abundance. Therefore, our analyses, which evaluate if population density (as a proxy for abundance) influences status, could be circular for species listed under these criteria. Nevertheless, simply excluding the aforementioned species would bias our sample by removing species that could be naturally rare, have distinct life-history and are all threatened. We addressed this problem by analysing the dataset including these species $(N=474)$ and the subset excluding the 21 species listed under criteria $\mathrm{C}$ and/or $\mathrm{D}(N=453)$ to evaluate if results were consistent.

To explore the role of brain mass on current vulnerability to extinction we defined the three scenarios mentioned in the introduction with several alternative models testing relationships between traits and vulnerability (Figs. 2, S2-S3). To reduce model space, links representing well-established relationships based on previous studies were included in all tested models. These relationships are represented by grey arrows in Figure 1b and include: $i$ ) the allometric relationship between brain mass and body mass (Harvey and Krebs 1990; Barton and Capellini 2011), ii) the links from gestation length 
to litter size and weaning age (Bielby et al. 2007), iii) the link between population density and extinction risk (Davidson et al. 2009; Fritz et al. 2009), and $i v$ ) the link between weaning age and extinction risk because weaning age is the only trait representing the reproductive output axis of the fast-slow life-history continuum (Bielby et al. 2007). Note that because all models include a causal link between body size and brain size, any association tested between brain size and life-history, ecology or vulnerability to extinction was tested accounting for allometric effects (akin to an ANCOVA model). In addition, previous work has shown that gestation length and weaning age are influenced by brain mass beyond allometric effects (Barton and Capellini 2011); thus, under the Brain and allometry scenario we assumed links from brain mass (but not body mass) to these two life-history traits and only tested the need for direct links from brain and/or body mass to population density and litter size (Fig. 2). Nevertheless, the possibility that gestation length and weaning age are more directly influenced by body mass than by brain mass was also tested, but in the Body mass allometry scenario. For each scenario we proposed distinct models that evaluate the need to include both direct links from traits that measure reproductive output (litter size and gestation length) to vulnerability to extinction as well as the need to include direct links from body and/or brain mass to vulnerability to extinction (Figs. 2, S2S3).

The proposed path models represent a series of hypothesized relationships between diverse traits and vulnerability to extinction based on theoretical and empirical evidence. However, the number of potential combinations of trait relationships within the Brain and allometry scenario is very large because, inevitably, under this scenario there are multiple paths by which brain and body mass could influence life-history and ecological traits (Fig. S4). On the other hand, the Brain costs and benefits and the Body mass allometry scenarios offer fewer combinations because they define unique and explicit links from brain and body mass to life-history and ecological traits (Figs S2-S3). To reduce model space for the Brain and allometry scenario we followed a two-step approach. We first identified the 
best model(s) among a set of alternatives describing how brain and body mass affect life-history and ecological traits (Fig. S4). The best model (or models, see model selection approach below) was subsequently used to test how these traits influence vulnerability to extinction, also allowing for direct effects of brain and body mass on vulnerability.

The minimal set of conditional independencies for each path model (von Hardenberg and Gonzalez-Voyer 2013) was tested using phylogenetic generalized least squares (PGLS) models (Martins and Hansen 1997) implemented using the package caper (Orme et al. 2012) in R. PGLS models have the advantage of incorporating different evolutionary models (e.g., Brownian motion, Ornstein-Uhlenbeck), combining categorical and continuous independent variables in a single analysis and estimating an evolutionary parameter $(\lambda)$ simultaneously with model fit that adjusts the variancecovariance matrix to adequately fit to the model of evolution, in our case a Brownian motion model (Freckleton et al. 2002; Revell 2010). The fit of a given path model to the data is estimated via the C statistic. The $\mathrm{C}$ statistic tests whether the minimum set of conditional independencies of a model is fulfilled by the observational data, thus it provides an estimate of the goodness of fit of the model to the data (Shipley 2013). A significant C statistic indicates that the model is a poor fit to the data (Shipley 2000). However, a limitation of the $\mathrm{C}$ statistic is that the fit of non-nested models cannot be compared. We therefore employed an information theoretical approach and compared the different path models using the C-statistic information criterion (analogous to the Akaike information criterion), CICc (Shipley 2013; von Hardenberg and Gonzalez-Voyer 2013). We calculated CIC weights for all models, which provide an estimate of the likelihood of each model (Burnham and Anderson 2002). When comparing models that could all be considered as supported based on $\mathrm{CICc}(\Delta \mathrm{CICc} \leq 2)$, we followed Burnham and Anderson (2002, p. 131) and generally ignored models that appeared supported by virtue of low $\triangle \mathrm{CICc}$ if they were more complex, nested versions of another supported model with minimal change in the estimate of goodness of fit, C statistic (see also Arnold 2010). Standardized path 
coefficients were calculated for all relationships in the best model using PGLS analyses on standardized variables (mean of 0 and standard deviation of 1 ).

Finally, to determine the robustness of our findings we tested whether results were consistent for different subsets of species, including separating species that are vulnerable to extinction due to different extrinsic factors (different paths to extinction, see Results) and those belonging to different taxonomic orders. We analysed the association between vulnerability to extinction and life-history traits, population density, and brain and body size for species threatened by different extrinsic factors using PGLS models (Martins and Hansen 1997). For the taxon-specific analyses we tested the bestsupported models for orders with sufficient data: Carnivora $(n=105$ species), Primates $(n=94$ species $)$ and Artiodactyla ( $n=60$ species). Rodents, while numerous ( $n=123$ species), were almost exclusively classified as least concern $(n=117)$ and thus, could be not evaluated separately.

\section{Results}

Analysing our complete dataset we did not find any evidence for direct, beneficial or detrimental, effects of brain size on vulnerability to extinction (Fig 1B). Nonetheless, our results indicated that enlarged brain size indirectly increases vulnerability to extinction, beyond allometric effects, because larger brains are associated with smaller litter sizes, longer gestation periods, and delayed weaning, and these last two traits are in turn associated with higher vulnerability to extinction (Fig. 1B). Body size also influences life-history and ecological traits via a direct link to litter size (which is also influenced by brain mass) and a direct link to population density which influences vulnerability to extinction (Fig. 1B). Among the 36 complete path models we proposed, we found those within the Brain and allometry scenario provided the best fit to the data ( $\triangle \mathrm{CICc}$ range: $0-7.58$; Table 1$)$. On the contrary, models that ignored the direct link between brain mass and life-history and ecological traits (Body mass allometry) provided a very poor fit to the data with values of $\Delta \mathrm{CICc}>57$ (Table S2). Models from the Brain costs 
and benefits scenario provided a better fit to the data than those in the Body mass allometry scenario but nonetheless received low support with $\triangle \mathrm{CICc}>15$ (Table S2). Note that only the path models in the Brain and allometry scenario presented non-significant $\mathrm{C}$ statistic values, indicating that all conditional independencies were met. On the contrary, models in the Body mass allometry and Brain costs and benefits scenario presented significant $\mathrm{C}$ statistic values (see Table S2). Results were qualitatively the same after excluding species listed as threatened under criteria $\mathrm{C}$ and D by the IUCN (relative to population abundance; Table S3, Fig. S5), which indicates that circularity was not an issue in our analyses.

When testing which model(s) best describe how brain and body mass affect life-history and ecological traits (the first step of the Brain and allometry scenario analyses) we found support for two models (Table S4). The model with the lowest CICc revealed direct links between brain mass and all three life-history traits (litter size, gestation length, and weaning age) as well as with population density, while also supporting a direct link from body mass to litter size and to population density (Table S4). The second supported model was a simpler version of the first, not including the link from brain mass to population density. All other models received low support $(\Delta \mathrm{CICc}>5)$. We present the results based on the simplest supported model from step 1 (not including a direct link from brain mass to population density). Nevertheless, results were qualitatively the same when using the model with the lowest CICc (Table S5, Fig. S6). Since we did not model any feedback from vulnerability to any of the traits, the definition of the relationships between traits (step 1) was not dependent on how these traits influenced vulnerability to extinction (step 2). Therefore, testing all possible combinations would not change the results, only unnecessarily increase the number of compared models.

Red List status as an ordinal variable 
Modelling Red List status as an ordinal variable under a phylogenetic Bayesian framework, we found the same qualitative results: lower population density $(\beta=-0.57,95 \% \mathrm{CI}=-0.92--0.28)$, longer gestation $(\beta=0.49,95 \% \mathrm{CI}=0.08-1.05)$ and delayed weaning $(\beta=0.51,95 \% \mathrm{CI}=0.16-0.81)$ are associated with greater vulnerability to extinction. Once we accounted for the effect of these life-history and ecological traits, there is no evidence for a direct association between brain mass $(\beta=0.41,95 \% \mathrm{CI}=-1.07-2.11)$ or body mass $(\beta=-0.23,95 \% \mathrm{CI}=-1.51-1.14)$ and vulnerability. Note that a model including life-history traits, and brain and body mass as predictors in the same model is equivalent to testing the conditional independencies in a path analysis framework, confirming the results above and supporting our finding that under current conditions large-brained mammals are generally more vulnerable to extinction.

\section{Vulnerability due to different extrinsic threats}

Previous studies have shown that there are different extrinsic factors that put species at risk, and that in light of these factors different intrinsic characteristics may make species more or less vulnerable to extinction (Owens and Bennett 2000; González-Suárez et al. 2013). A recent study described two main paths to extinction in mammals based on the apparent accumulation of extrinsic threats (GonzálezSuárez and Revilla 2014). The first path, which affects mostly large, widespread species, starts with their direct exploitation (including all types of harvest) followed by habitat degradation. The second path, which affects smaller mammals with narrower distribution ranges, is defined by an intensification of human land uses leading to habitat loss and degradation but does not involve direct exploitation. Because how intrinsic traits influence vulnerability to extinction could depend on the main threatening factors, we evaluated whether our results are consistent when analysing separately groups of species following different paths to extinction (i.e., affected by different types of threats). Considering the descriptions of threats provided by the IUCN (2010) we found that of the 474 species in our dataset 201 species have no listed threats. From the remainder, 228 appear to follow the first path (affected by 
direct exploitation), 40 species follow the second path to extinction (being affected by activities that lead to habitat loss and degradation but not by direct exploitation) and 5 species have other threat combinations (idiosyncratic paths as in González-Suárez and Revilla 2014).

For the 228 species in the exploitation path, we found that longer gestation periods and delayed weaning (which are influenced by brain size) are also associated with a significant increase in vulnerability to extinction (PGLS $\lambda=0.20$; gestation length: $\beta \pm \mathrm{SE}=0.83 \pm 0.379, \mathrm{p}=0.030$; weaning age: $\beta \pm S E=0.64 \pm 0.295, p=0.031)$. However, we found no significant association between population density and vulnerability to extinction $(\beta \pm \mathrm{SE}=-0.13 \pm 0.091, \mathrm{p}=0.167)$ when controlling for phylogenetic relatedness. Controlling for these three traits, body mass and brain mass were not directly associated with vulnerability to extinction (body mass: $\beta \pm \mathrm{SE}=0.42 \pm 0.337, \mathrm{p}=0.208$; brain mass: $\beta \pm \mathrm{SE}=-$ $0.91 \pm 0.571, \mathrm{p}=0.110$ ). These exploited species live at generally low population densities (median population density is $3 \mathrm{ind} / \mathrm{km}^{2}$ compared to $18 \mathrm{ind} / \mathrm{km}^{2}$ for the complete dataset) and apparently their risk of extinction is influenced more by their reproductive traits than their ecology (i.e. population density). Thus, in harvested species a slower life-history increases vulnerability, potentially due to reduced capability to counteract the negative effects of the additional extrinsic mortality resulting from hunting.

Among the small subset of species $(\mathrm{N}=40)$ that appear to follow the habitat loss path to extinction, only 11 are classified as threatened (vulnerable, endangered, or critically endangered). For these 40 species we found no significant relationship between any of the life-history or ecological traits and vulnerability to extinction (PGLS, $\lambda=0.0$; gestation: $\beta \pm S E=-0.92 \pm 0.588, p=0.125$; weaning: $\beta \pm S E=0.46 \pm 0.621, p=0.467$; population density: $\beta \pm S E=-0.05 \pm 0.201, p=0.820$ ), when controlling for phylogenetic relatedness. Controlling for these three traits, body mass and brain mass were not directly 
associated with vulnerability to extinction (body mass: $\beta \pm \mathrm{SE}=-0.95 \pm 0.843, \mathrm{p}=0.261$; brain mass: $\beta \pm S E=0.74 \pm 1.295, p=0.570)$. Additional data would be necessary to determine if this lack of significant relationships is due to the very limited sample size or in fact species affected primarily by habitat loss and degradation do have different intrinsic traits influencing their vulnerability.

\section{Taxon-specific analyses}

When fitting the three best-supported models for the overall dataset to Primates, Carnivora and Artiodactyla separately, we found some conditional independencies were not met for these groups. We made minor changes to define the relationships between brain size, body size, population density and life-history traits generating order-specific models that present minor, qualitative differences with those for the entire dataset (for details see Supplementary Results, Figure S7). Whether these differences could reflect biologically relevant differences among orders is an intriguing question, but one beyond the scope of the present study which focuses on vulnerability to extinction. As with the overall dataset, these order-specific analyses indicate that increased brain size is indirectly associated with a higher vulnerability to extinction due to longer gestation and delayed weaning (Table S6). For Primates and Carnivora, larger brain size is also associated with lower population density, which in turn increases vulnerability (Table S6). Interestingly, for Primates the best model also included a direct positive link between brain size and vulnerability to extinction, beyond the effects of gestation, weaning and population density. This result indicates that among Primates, larger brains are directly, as well as indirectly, associated with increased vulnerability to extinction.

\section{Discussion}

Our results showed that larger brain size does not provide net benefits for mammals facing current threats. On the contrary, the path models which best fitted our data indicate that relatively larger 
brains reduce the potential population growth rate and thus, indirectly, increase vulnerability to extinction, because larger brains are associated with longer gestation periods and delayed weaning which in turn, are associated with increased risk of extinction. We find that at present, the behavioural plasticity that can be associated with increased brain mass is apparently not sufficient to compensate for the costs of developing and maintaining a larger brain, and thus does not confer sufficient net benefits to mammals in the face of current anthropogenic threats. The results are unchanged when modelling the response as an ordinate variable, when excluding species classified as vulnerable due to their population size, and when considering different paths to extinction. Separate analyses for the three orders for which sufficient data were available (Primates, Carnivora and Artiodactyla) also confirm our finding: larger brain size indirectly increases vulnerability due to longer gestation periods and delayed weaning. Interestingly, for primates larger brain size is also directly associated with increased vulnerability to extinction. These results suggest that conservation measures should particularly target large-brained primate species due to their higher intrinsic vulnerability to extinction and point to another distinction between humans and non-human primates. While enlarged brain size likely played a key role in the successful invasion of virtually all continents by humans, enlarged brain size makes non-human primates more vulnerable.

An alternative explanation to our results is that brain size is simply a better estimator of general size and thus, shows a stronger correlation with life-history traits than body mass (Harvey and Krebs 1990). Body mass is a more variable trait than brain mass, both temporally for a given individual as well as among individuals. It is unlikely, however, that the difference in precision between estimates of these two traits could explain our results. First, the variation in body mass of the species included in our analyses (spanning six orders of magnitude) should minimize the potential noise due to intraspecific variation in size. Second, models ignoring direct relationships between brain mass and life-history traits provided a particularly poor fit to the data; in fact all such models were rejected based on the value of 
the C-statistic (Table S2) yet ignoring body mass also resulted in poor models. The best-supported models included effects of both brain and body mass suggesting both variables are not simply different estimates of total size but describe distinct aspects of allometric and energetic relationships.

Our results offer insights into the aforementioned distinct allometric and energetic associations, revealing interesting relationships between brain and body mass and life-history and ecological traits and highlight the advantage of using phylogenetic path analysis to disentangle these complex relationships. Firstly, our analyses suggest independent associations of brain and body mass with litter size. The influence of body mass on litter size may reflect physical limitations associated with the intrauterine space available for the neonates, while the influence of brain size on litter size could reflect energetic costs associated with enlarged brain mass and larger neonate size (Barton and Capellini 2011). Our results also suggest that brain size plays a key role determining gestation length and weaning age, beyond allometric effects, as models that did not include direct links between brain mass and gestation length or weaning age (Body mass allometry scenario) provided a poor fit to the data. Employing phylogenetic path analysis, our results allow us to propose that the observed correlations between body size and gestation length and weaning age are a result of the allometric relationship between brain size and body size rather than direct causal links (allometry indirectly influences gestation length and weaning age because all models included a causal link between body size and brain size). These results are in accordance with the findings of Barton and Capellini (2011) who suggested that longer gestation periods and delayed weaning are a direct result of enlarged brain size, possibly due to increased energetic costs of development at the foetal and juvenile stages. In addition, our results show that body size influences population density, as expected larger species require more space per individual and thus live at lower densities. More interestingly, our analyses also suggest the possibility of an additional link between brain mass and population density, particularly supported for Carnivora and Primates, which could reflect a reduction in a given area's carrying capacity due to the 
energetic costs of enlarged brains (Isler and van Schaik 2009b). As far as we know a direct link between brain mass and population density has not been previously explicitly proposed and is a hypothesis that will need to be explored further.

Relative brain size has increased during the evolutionary history of vertebrates presumably because larger brains conferred an evolutionary advantage (Rowe et al. 2011). Nevertheless, our results indicate that under current conditions large brains increase vulnerability to extinction for mammals. This relationship has also been reported by a recent study (Abelson 2016) that correlated the probability of being threatened (transformed to a binary variable) in mammals with relative brain sizes, estimated as residuals of a relationship between brain size and body size (for criticisms of this approach see García-Berthou 2001; Freckleton et al. 2002). However, the aforementioned work ignored other intrinsic traits previously found to correlate with vulnerability and brain size. Exploring multiple intrinsic factors simultaneously in a flexible path analyses approach our results show that the correlation is not due to a direct association between brain size and vulnerability, but rather it is due to the association between brain size and life-history, which in turn is associated with vulnerability to extinction. Our approach allowed us to disentangle direct from indirect associations between variables to gain a better understanding of the complex associations between intrinsic traits and vulnerability to extinction.

Interestingly, our results of a negative effect of brain size in vulnerability contrasts with findings for birds where large brains apparently do not increase extinction risk (Nicolakakis et al. 2003). The discrepancy in the results between mammals and birds could be explained by the fact that in altricial birds enlarged brain size is not associated with a reduction in reproductive output because of allomaternal care of offspring (Isler and van Schaik 2009a). Many of the threats affecting mammals today are the result of human actions. Rapid environmental change intensifies the risk of extinction 
because populations are unable to adapt quickly enough (Lindsey et al. 2013). Moreover, widespread harvesting of many species has likely increased the energetic and reproductive costs of a large brain because species need to increase their reproduction rate to compensate for the increased mortality. However, species with large brains have slow reproduction rates that cannot be easily hastened. By reducing intrinsic growth rates, larger brains may currently be imposing demographic constraints that translate into higher vulnerability contrary to the selective forces that during millions of years have favoured increased brain size to the diversity that we observe today. In this respect, our results indicating that increased brain size indirectly increases vulnerability, by slowing-down the life-history, are counter to the cognitive buffer hypothesis, which proposes that larger brains facilitate the construction of behavioural responses to novel or complex challenges resulting in increased survival rates and prolonged longevity (Sol 2009).

Certainly, under particular conditions large brains still appear to confer an advantage for some species (Sol et al. 2007; Sol et al. 2008; Maklakov et al. 2011). As mentioned above, humans are likely the best example of the advantages of having a large brain. However, our study shows that these benefits are not general. Our results highlight the importance of taking into consideration the higher vulnerability of large-brained species due to their reduced potential population growth rate when implementing species-specific conservation plans. Our sample includes approximately $10 \%$ of all extant mammalian species and most taxonomic orders (Fig. S1), however some groups (carnivores, artiodactyls and primates) are over-represented while others (rodents and bats) are under-represented. As more data becomes available our findings could be reevaluated to explore if brain size has different costs and benefits among these less-studied groups. In the meantime our analyses indicate that in today's world the once beneficial large brain has apparently become a burden for many mammals. 
Acknowledgements: We thank A. von Hardenberg, M. Delibes, B. Van Valkenburg, N. Kolm, the members of the Conservation and Evolutionary Genetics group at the Estación Biológica de Doñana (EBD-CSIC, Spain) for comments and discussions. Andrea Barón helped us gather data from the literature. For comments on an earlier version we thank Dr Ruth Shaw, Dr Jeffrey Good and three anonymous reviewers. AG-V was funded by a Junta para la Ampliación de Estudios (JAEDOC030) post-doctoral fellowship, co-funded by the European Union, and by a Young Researcher grant from the Swedish Research Council (Vetenskapsrådet). MG-S was funded by the European Community’s Seventh Framework Programme (FP7/2007-2013) under grant agreement n 235897 and by a Juan de la Cierva post-doctoral fellowship (JCI-2011-09158). This work was funded by the Spanish Ministry of Science and Innovation (CGL2010-21250, CGL2013-47547, CGL2009-07301 and CGL2012-35931 co-funded by FEDER) and Proyecto de Captación del Conocimiento para Andalucía (CV).

Statement of authorship: AG-V and MG-S conceived the study. AGV performed the phylogenetic comparative analyses. MG-S gathered the data and prepared the figures. AG-V and MG-S drafted the manuscript. All authors designed the study, interpreted the results and contributed to the final version of the paper.

Data accessibility: Data available from the Dryad Digital Repository: http://dx.doi.org/10.5061/dryad.m2tj3. 


\section{References}

Abelson, E. S. 2016. Brain size is correlated with endangerment status in mammals. Proceedings of the Royal Society of London B: Biological Sciences 283.

Aiello, L. C. and P. Wheeler. 1995. The expensive-tissue yypothesis: the brain and the digestive system in human and primate evolution. Cur. Anthropol. 36:199-221.

Amiel, J. J., R. Tingley, and R. Shine. 2011. Smart moves: effects of relative brain size on establishment success of invasive amphibians and reptiles. PLoS ONE 6:e18277.

Arnold, T. W. 2010. Uninformative parameters and model selection using Akaike's Information Criterion. The Journal of Wildlife Management 74:1175-1178.

Barnosky, A. D., N. Matzke, S. Tomiya, G. O. U. Wogan, B. Swartz, T. B. Quental, C. Marshall, J. L. McGuire, E. L. Lindsey, K. C. Maguire, B. Mersey, and E. A. Ferrer. 2011. Has the Earth's sixth mass extinction already arrived? Nature 471:51-57.

Barton, R. A. and I. Capellini. 2011. Maternal investment, life histories, and the costs of brain growth in mammals. Proc. Natl. Acad. Sci. USA 108:6169-6174.

Bennett, P. M. and I. P. F. Owens. 1997. Variation in extinction risk among birds: chance or evolutionary predisposition? Proc Biol Sci 264:401-408.

Bielby, J., G. M. Mace, O. R. P. Bininda-Emonds, M. Cardillo, J. L. Gittleman, K. E. Jones, C. D. L. Orme, and A. Purvis. 2007. The fast-slow continuum in mammalian life history: an empirical reevaluation. Am. Nat. 169:748-757.

Bininda-Emonds, O. R. P., M. Cardillo, K. E. Jones, R. D. E. MacPhee, R. M. D. Beck, R. Grenyer, S. A. Price, R. A. Vos, J. L. Gittleman, and A. Purvis. 2007. The delayed rise of present-day mammals. Nature 446:507-512.

Bodmer, R. E., J. F. Eisenberg, and K. H. Redford. 1997. Hunting and the likelihood of extinction of Amazonian mammals. Cons. Bio. 11:460-466. 
Boyer, A. G. 2010. Consistent ecological selectivity through time in Pacific island avian extinctions. Cons. Bio. 24:511-519.

Burnham, K. P. and D. R. Anderson. 2002. Model selection and multimodel inference. A practical information-theoretic approach. Springer, New York, USA.

Cardillo, M., G. M. Mace, J. L. Gittleman, K. E. Jones, J. Bielby, and A. Purvis. 2008. The predictability of extinction: biological and external correlates of decline in mammals. Proc. Roy. Soc. B 275:1441-1448.

Cardillo, M., G. M. Mace, J. L. Gittleman, and A. Purvis. 2006. Latent extinction risk and the future battlegrounds of mammal conservation. Proc. Natl. Acad. Sci. USA 103:4157-4161.

Cardillo, M., G. M. Mace, K. E. Jones, J. Bielby, O. R. P. Bininda-Emonds, W. Sechrest, C. D. L. Orme, and A. Purvis. 2005. Multiple causes of high extinction risk in large mammal species. Science 309:1239-1241.

Cardillo, M., A. Purvis, W. Sechrest, J. L. Gittleman, J. Bielby, and G. M. Mace. 2004. Human population density and extinction risk in the world's carnivores. PLoS Biology 2:909-914. Davidson, A. D., A. G. Boyer, H. Kim, S. Pompa-Mansilla, M. J. Hamilton, D. P. Costa, G. Ceballos, and J. H. Brown. 2012. Drivers and hotspots of extinction risk in marine mammals. Proc. Natl. Acad. Sci. USA 109:3395-3400.

Davidson, A. D., M. J. Hamilton, A. G. Boyer, J. H. Brown, and G. Ceballos. 2009. Multiple ecological pathways to extinction in mammals. Proc. Natl. Acad. Sci. USA 106:10702-10705.

Foufopoulos, J. and A. R. Ives. 1999. Reptile extinctions on land-bridge islands: Life-history attributes and vulnerability to extinction. Am. Nat. 153:1-25.

Freckleton, R. P., P. H. Harvey, and M. Pagel. 2002. Phylogenetic analysis and comparative data: a test and review of evidence. Am. Nat. 160:712-726.

Fritz, S. A., O. R. P. Bininda-Emonds, and A. Purvis. 2009. Geographical variation in predictors of mammalian extinction risk: big is bad, but only in the tropics. Ecol. Lett. 12:538-549. 
García-Berthou, E. 2001. On the misuse of residuals in ecology: testing regression residuals vs. the analysis of covariance. J. Anim. Ecol. 70:708-711.

González-Lagos, C., D. Sol, and S. M. Reader. 2010. Large-brained mammals live longer. J. Evol. Biol. 23:1064-1074.

González-Suárez, M., A. Gómez, and E. Revilla. 2013. Which intrinsic traits predict vulnerability to extinction depends on the actual threatening processes. Ecosphere 4:76.

González-Suárez, M., P. M. Lucas, and E. Revilla. 2012. Biases in comparative analyses of extinction risk: mind the gap. J. Anim. Ecol. 81:1211-1222.

González-Suárez, M. and E. Revilla. 2013. Variability in life-history and ecological traits is a buffer against extinction in mammals. Ecol. Lett. 16:242-251.

González-Suárez, M. and E. Revilla. 2014. Generalized drivers in the mammalian endangerment process. PLoS ONE 9:e90292.

Gonzalez-Voyer, A. and A. von Hardenberg. 2014. Introduction to phylogenetic path analysis. Pp. 201229 in L. Z. Garamszegi, ed. Modern phylogenetic comparative methods and their application in evolutionary biology. Springer-Verlag, Berlin.

Hadfield, J. D. 2010. MCMC methods for multi-response generalized linear mixed models: the MCMCglmm R package. Journal of Statistical Software 33:1-22.

Harvey, P. H. and J. R. Krebs. 1990. Comparing brains. Science 249:140-146.

Isler, K. and C. van Schaik. 2009a. Why are there so few smart mammals (but so many smart birds)? Biol. Lett. 5:125-129.

Isler, K. and C. van Schaik. 2009b. The expensive brain: a framework for explaining evolutionary changes in brain size. J. Hum. Evol. 57:392-400.

IUCN. 2010. Red List of Threatened Species. Version 2010.4.

Jones, K. E., J. Bielby, M. Cardillo, S. A. Fritz, J. O'Dell, C. D. L. Orme, K. Safi, W. Sechrest, E. H. Boakes, C. Carbone, C. Connolly, M. J. Cutts, J. K. Foster, R. Grenyer, M. Habib, C. A. Plaster, S. A. 
Price, E. A. Rigby, J. Rist, A. Teacher, O. R. P. Bininda-Emonds, J. L. Gittleman, G. M. Mace, A. Purvis, and W. K. Michener. 2009. PanTHERIA: a species-level database of life history, ecology, and geography of extant and recently extinct mammals. Ecology 90:2648-2648.

Jones, K. E., A. Purvis, and J. L. Gittleman. 2003. Biological correlates of extinction risk in bats. Am. Nat. 161:601-614.

Kotrschal, A., B. Rogell, A. Bundsen, B. Svensson, S. Zajitschek, I. Bränström, S. Immler, A.

Maklakov, and N. Kolm. 2013. Artificial selection on relative brain size in the guppy reveals costs and benefits of evolving a larger brain. Current Biology 23:168-171.

Lefebvre, L., S. M. Reader, and D. Sol. 2004. Brains, innovations and evolution in birds and primates. Brain Behav. Evol. 63:233-246.

Lindsey, H. A., J. Gallie, S. Taylor, and K. Benjamim. 2013. Evolutionary rescue from extinction is contingent on a lower rate of environmental change. Nature 494:463-467.

Maklakov, A., S. Immler, A. Gonzalez-Voyer, J. Rönn, and N. Kolm. 2011. Brains and the city: bigbrained passerine birds succeed in urban environments. Biol. Lett. 7:730-732.

Martins, E. P. and T. F. Hansen. 1997. Phylogenies and the comparative method: a general approach to incorporating phylogenetic information into the analysis of interspecific data. Am. Nat. 149:646-667. Montgomery, S. H., I. Capellini, R. A. Barton, and N. I. Mundy. 2010. Reconstructing the ups and downs of primate brain evolution: implications for adaptive hypotheses and Homo floresiensis. BMC Biology 8:1-19.

Murray, K. A., D. Rosauer, H. McCallum, and L. F. Skerratt. 2011. Integrating species traits with extrinsic threats: closing the gap between predicting and preventing species declines. Proc Biol Sci 278:1515-1523.

Nicolakakis, N., D. Sol, and L. Lefebvre. 2003. Behavioural flexibility predicts species richness in birds, but not extinction risk. Anim. Behav. 65:445-452. 
Orme, C. D. L., R. P. Freckleton, G. R. Thomas, T. Petzoldt, S. A. Fritz, N. Isaac, and W. Pearse. 2012. caper: Comparative Analyses of Phylogenetics and Evolution in R. R package version 0.5.

Owens, I. P. F. and P. M. Bennett. 2000. Ecological basis of extinction risk in birds: Habitat loss versus human persecution and introduced predators. Proc. Natl. Acad. Sci. USA 97:12144-12148.

Plummer, M., N. Best, K. Cowles, and K. Vines. 2006. CODA: Convergence Diagnosis and Output Analysis for MCMC. R News 6:7-11.

Pocock, M. J. O. 2011. Can traits predict species' vulnerability? A test with farmland passerines in two continents. Proc Biol Sci 278:1532-1538.

Purvis, A., P. M. Agapow, J. L. Gittleman, and G. M. Mace. 2000a. Nonrandom extinction and the loss of evolutionary history. Science 288:328-330.

Purvis, A., J. L. Gittleman, G. Cowlishaw, and G. M. Mace. 2000b. Predicting extinction risk in declining species. Proceedings of the Royal Society of London B Biological Sciences 267:1947-1952. R Development Core Team. 2011. R: A language and environment for statistical computing. R Foundation for Statistical Computing, Vienna, Austria.

Reader, S. M. and K. E. Laland. 2002. Social intelligence, innovation, and enhanced brain size in primates. Proc. Natl. Acad. Sci. USA 99:4436-4441.

Revell, L. J. 2010. Phylogenetic signal and linear regression on species data. Meth. Ecol. Evol. 1:319329.

Rowe, T. B., T. E. Macrini, and Z.-X. Luo. 2011. Fossil evidence on origin of the mammalian brain. Science 332:955-957.

Shipley, B. 2000. Cause and correlation in biology: A user's guide to path analysis, structural equations and causal inference. Cambridge University Press, Cambridge.

Shipley, B. 2013. The AIC model selection method applied to path analytic models compared using a d-separation test. Ecology 94:560-564. 
Shultz, S., R. B. Bradbury, K. L. Evans, R. D. Gregory, and T. M. Blackburn. 2005. Brain size and resource specialization predict long-term population trends in British birds. Proc Biol Sci 272:23052311.

Sol, D. 2009. Revisiting the cognitive buffer hypothesis for the evolution of large brains. Biol. Lett. 5:130-133.

Sol, D., S. Bacher, S. M. Reader, and L. Lefebvre. 2008. Brain size predicts the success of mammal species introduced into novel environments. Am. Nat. 172:S63-S71.

Sol, D., T. Székely, A. Liker, and L. Lefebvre. 2007. Big-brained birds survive better in nature. Proc Biol Sci 274:763-769.

Sol, D., S. Timmermans, and L. Lefebvre. 2002. Behavioural flexibility and invasion success in birds. Anim. Behav. 63:495-502.

Striedter, G. F. 2005. Principles of brain evolution. Sinauer Associates Inc, Sunderland, MA. Urban, M. C. 2015. Accelerating extinction risk from climate change. Science 348:571-573. Verde Arregoitia, L. D. 2016. Biases, gaps, and opportunities in mammalian extinction risk research. Mammal Review 46:17-29. von Hardenberg, A. and A. Gonzalez-Voyer. 2013. Disentangling evolutionary cause-effect relationships with phylogenetic confirmatory path analysis. Evolution 67:378-387. 
Table 1. Ranking of the best path models tested based on CICc values. Model codes correspond to diagrams presented in Fig. 2. All of the models included in the table are from the Brain and Allometry scenario. For each model we report the $\mathrm{CICc}$ value, $\Delta \mathrm{CICc}$ value, $\mathrm{CICc}$ weights $(\omega)$, C-statistic $(\mathrm{C})$. Models were based on 474 mammalian species (Fig. S1). We present here only models with $\Delta \mathrm{CICc}<10$. Results from all tested models are given in Table S2.

\begin{tabular}{lllll}
\hline Model & CICc & $\Delta$ CICc & $\omega$ & C \\
\hline AB2 & 57.87 & 0.00 & 0.31 & 20.37 \\
AB5 & 59.29 & 1.42 & 0.15 & 19.62 \\
AB8 & 59.56 & 1.69 & 0.13 & 19.89 \\
AB1 & 59.73 & 1.86 & 0.12 & 20.06 \\
AB11 & 60.76 & 2.89 & 0.07 & 18.90 \\
AB4 & 61.15 & 3.28 & 0.06 & 19.30 \\
AB7 & 61.43 & 3.55 & 0.05 & 19.57 \\
AB3 & 62.56 & 4.69 & 0.03 & 25.06 \\
AB10 & 62.69 & 4.82 & 0.03 & 18.65 \\
AB9 & 64.49 & 6.62 & 0.01 & 24.82 \\
AB6 & 64.62 & 6.75 & 0.01 & 24.95 \\
AB12 & 65.46 & 7.58 & 0.01 & 23.60 \\
\hline
\end{tabular}




\section{A) Conceptual framework}

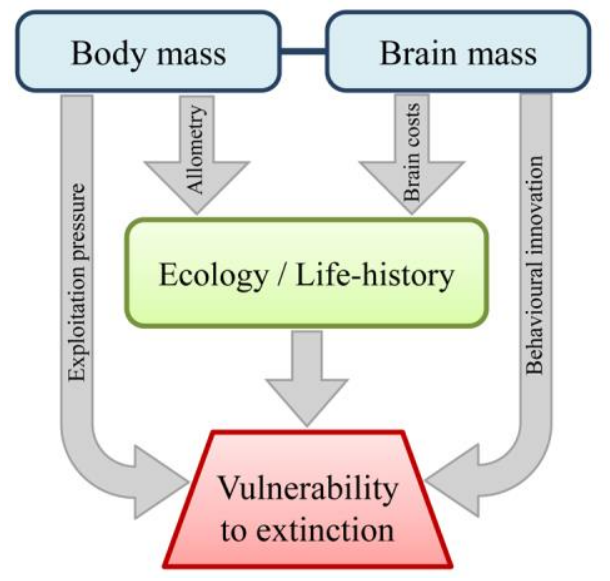

\section{B) Path analyses results}

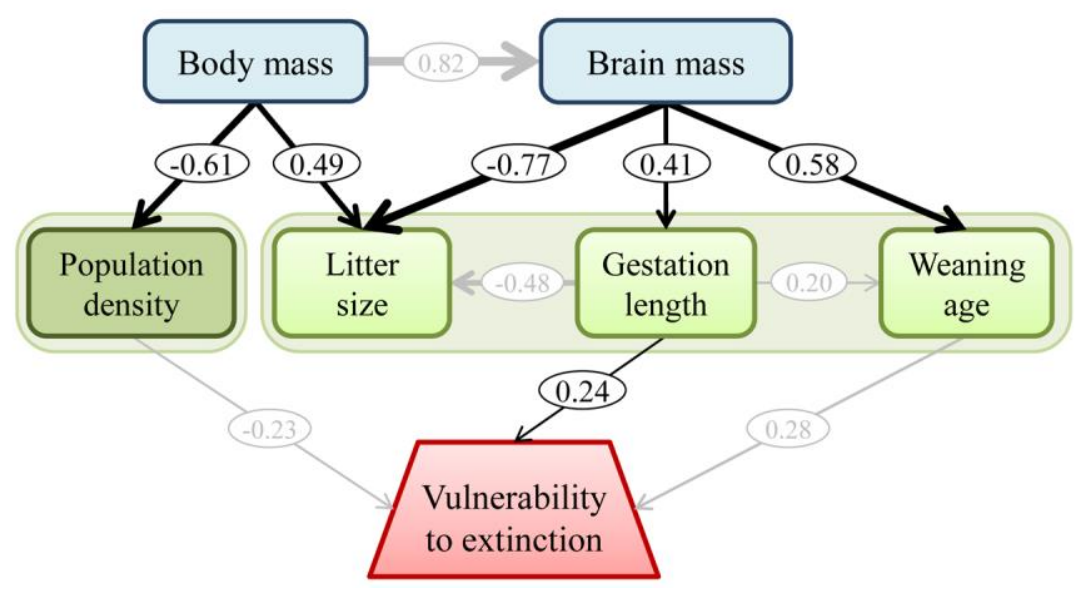

Figure 1. Hypothesized and tested relationships among body mass, brain mass, life history, ecology, and vulnerability to extinction in extant mammals. A) Conceptual framework describing the hypotheses tested in this study. B) Path diagram showing the empirical relationships described by the model best supported by the data (Table 1). The width of the arrows reflects the value of the standardized slope coefficient (higher values - wider arrows), and the numbers represent the value of the standardized slope coefficients. Grey arrows indicate relationships that were included in all models. 


\section{Allometry and brain}

Do both traits that reflect reproductive output directly influence status?
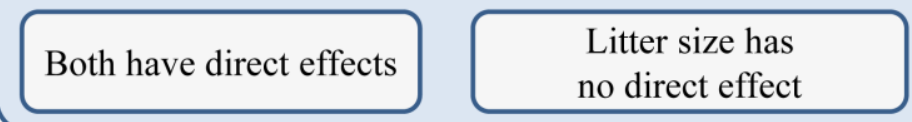

Gestation has

Both have direct effects
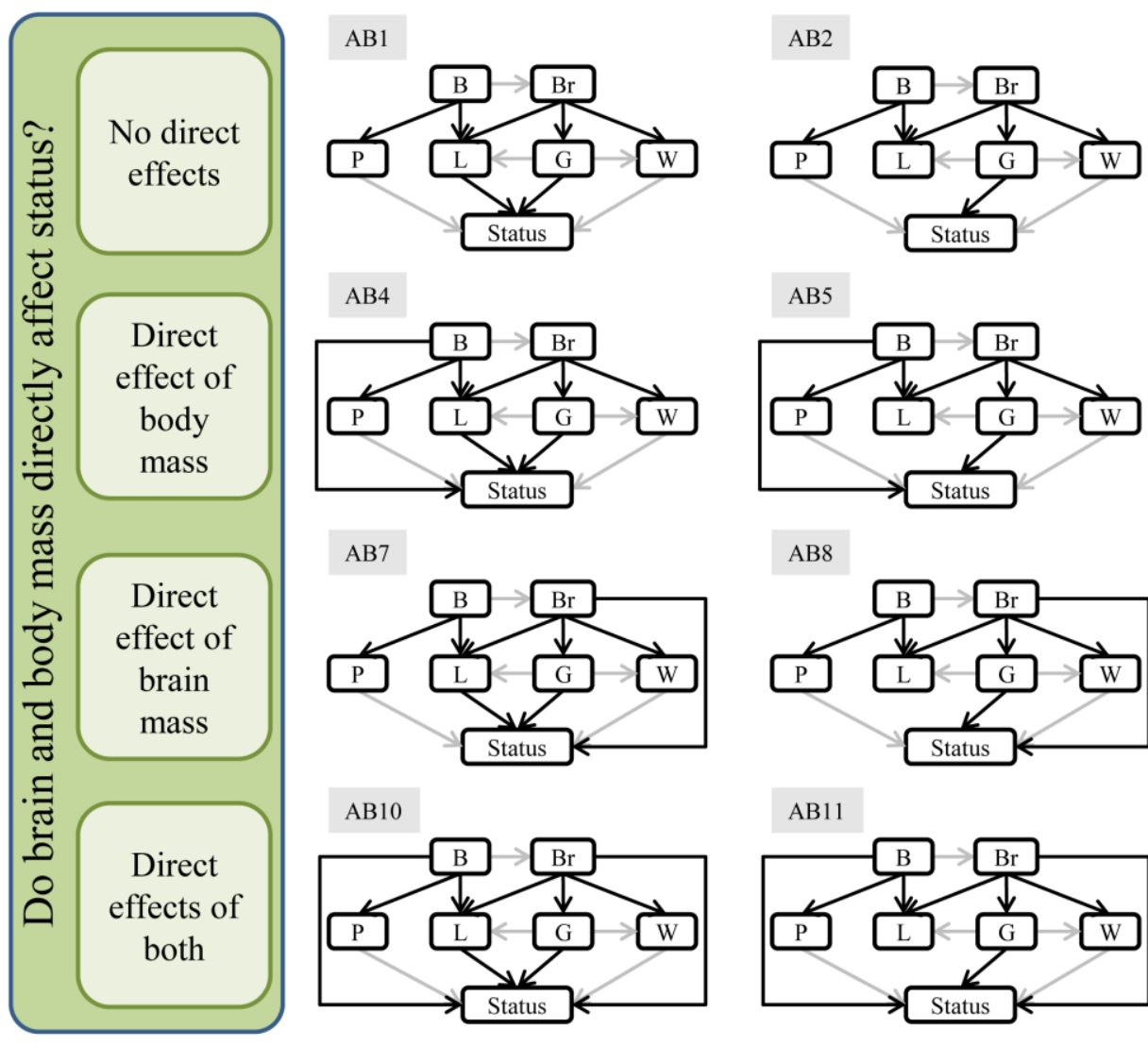

AB5
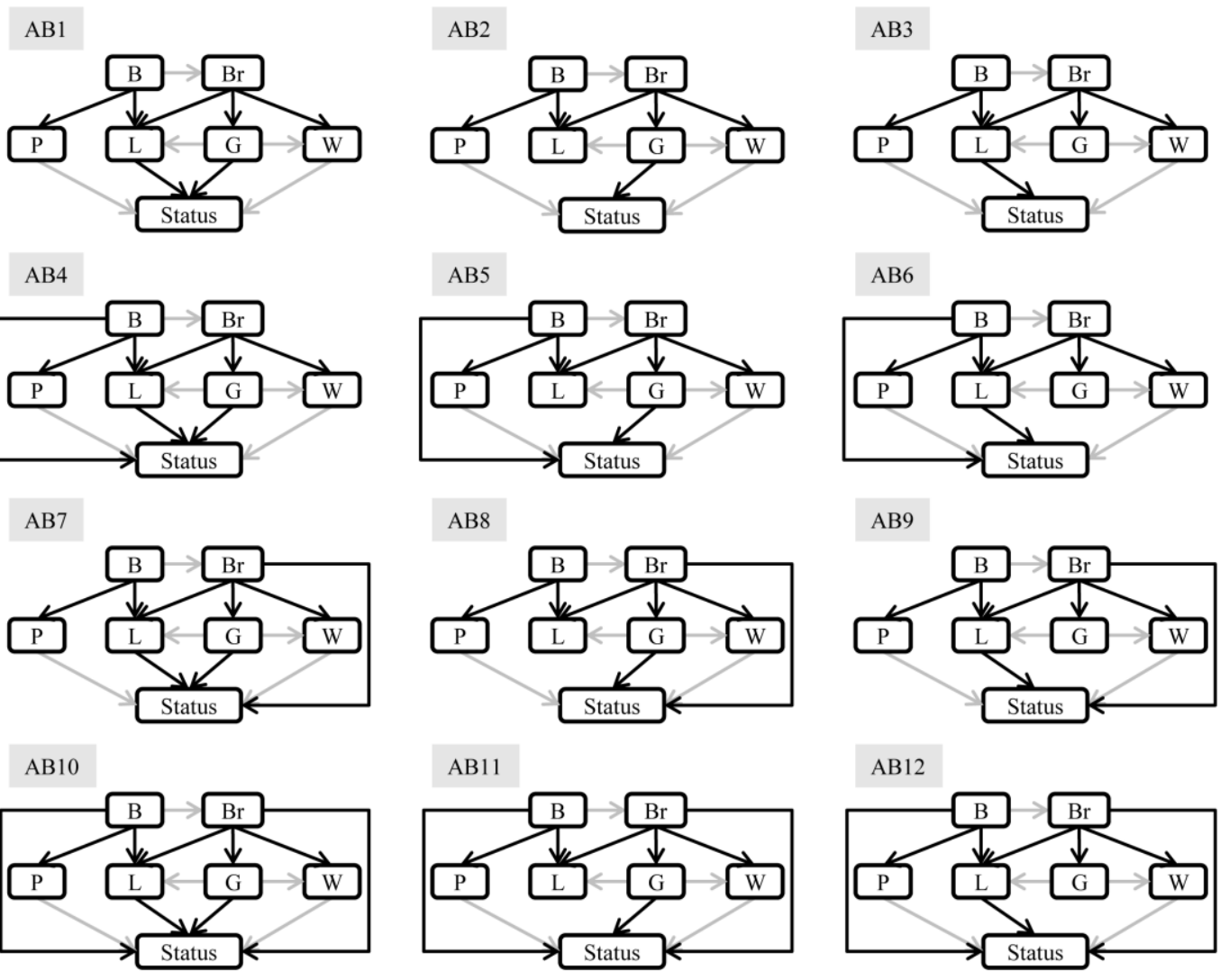

AB9

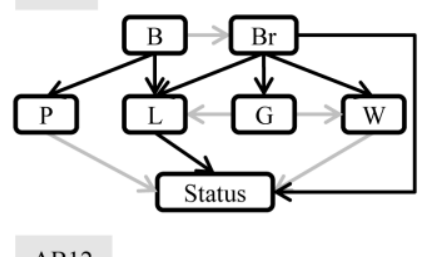

AB12

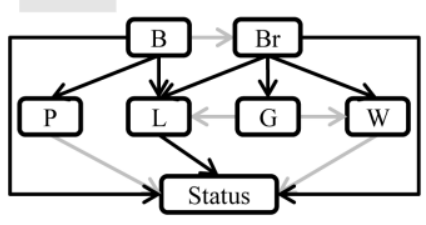

Figure 2. Path models tested under the Brain and allometry scenario, which proposes that a

combination of allometric effects and energetic costs of brain mass influence life-history and ecological traits. Body mass (B), brain mass (Br), litter size (L), gestation length (G), weaning age (W), population density (P), and vulnerability to extinction based on the IUCN Red List categories (Status). Grey arrows indicate relationships that were included in all models. 


\section{Supporting Information for}

Larger brain size indirectly increases vulnerability to extinction in mammals.

by A. Gonzalez-Voyer, M. González-Suárez, C. Vilá and E. Revilla

\section{Supplementary Methods}

To identify species' traits that have been consistently associated with vulnerability to extinction (defined by the IUCN Red List status) we conducted a literature review in the fall of 2013. We searched for global comparative studies that aimed to identify the key intrinsic factors that influenced vulnerability to extinction in mammals. We selected studies searching for correlates of vulnerability, excluding studies that focused on the role of particular traits (and thus, only tested a very limited subset of species' traits).

We located ten studies published from 2000 to 2013 from which we gathered information on which traits were analyzed and revealed as consistently significant or relevant for vulnerability (Table S1). Relevance was scored as 0 if the trait was tested but not identified as significant or selected in any tested models, 1 if the trait was significant or selected in only some models, or 2 if the trait was significant or selected in all models or the model selected as "best" by the authors. Based on these scores from the 10 studies we then calculated a total trait score (the sum of all scores) to represent overall trait importance.

For our analyses we selected one morphological and one ecological trait. For traits related to reproductive performance we selected those representing the distinct aspects of the slow-fast continuum that had the highest scores and the greatest amount of available data. While the focus was on selected traits from the slow-fast continuum we show results from all traits analysed by the revised studies.

Table S1. Results from 10 global comparative studies of vulnerability to extinction in mammals summarized for each traits as: 0 (trait was evaluated but not identified as relevant), 1 (trait evaluated and identified as relevant in at least one analysis; traits could be identified as relevant for subsets of the data or only in certain combinations of predictors), 2 (trait identified as relevant in all analyses or the model selected as "best" by the authors), and dash (-) to indicate a trait not evaluated in that study. Most studies analyzed mammalian biodiversity in general (with limitations based on available data), except for reference 1 (limited to Carnivora and Primates), reference 2 (Chiroptera), reference 3 (Carnivora), and reference 9 (marine mammals). In addition we report the number of species for which data were available (when analyses were based on different sample sizes we report the largest sample considered to reflect all species that were evaluated) and the total number of evaluated traits (intrinsic traits considered in at least one analysis). The traits selected for this present study are highlighted in bold. The complete reference information is provided below.

\begin{tabular}{|c|c|c|c|c|c|c|c|c|c|c|c|c|c|}
\hline \multirow[t]{2}{*}{ Evaluated traits } & \multicolumn{10}{|c|}{ References } & \multirow{2}{*}{$\begin{array}{l}\text { Trait } \\
\text { score }\end{array}$} & \multirow{2}{*}{$\begin{array}{l}\text { Times } \\
\text { tested }\end{array}$} & \multirow{2}{*}{$\begin{array}{c}\text { Data } \\
\text { available }\end{array}$} \\
\hline & [1] & {$[2]$} & [3] & [4] & {$[5]$} & {$[6]$} & [7] & {$[8]^{*}$} & [9] & {$[10]$} & & & \\
\hline \multicolumn{14}{|l|}{ Morphological } \\
\hline Adult body mass & 2 & 1 & $\mathbf{0}$ & 2 & 2 & 1 & 2 & 2 & 2 & 2 & 16 & 10 & 590 \\
\hline Neonate body mass & - & - & - & 1 & - & 0 & - & - & - & 1 & 2 & 3 & 515 \\
\hline Weanling body mass & - & - & - & - & - & - & - & - & 2 & - & 2 & 1 & 284 \\
\hline Aspect ratio & - & 2 & - & - & - & - & - & - & - & - & 2 & 1 & 0 \\
\hline Adult body length & - & - & - & - & - & - & - & - & - & 1 & 1 & 1 & 472 \\
\hline Adult forearm length & - & - & - & - & - & - & - & - & - & 1 & 1 & 1 & 33 \\
\hline Teat number & - & - & - & - & - & - & - & - & - & 0 & 0 & 1 & 198 \\
\hline \multicolumn{14}{|l|}{ Ecological } \\
\hline Geographic range & 2 & 2 & 2 & 2 & 2 & 2 & 2 & 2 & 2 & 2 & 20 & 10 & 592 \\
\hline Pop density & 2 & - & 2 & 2 & 1 & 2 & 2 & 2 & - & 2 & 15 & 8 & 409 \\
\hline Group size/Sociality & 0 & 1 & 0 & - & - & 0 & 2 & - & 2 & 1 & 6 & 7 & 228 \\
\hline Trophic level/Diet & 2 & - & 2 & - & - & 0 & 1 & - & 1 & - & 6 & 5 & 515 \\
\hline Habitat mode & - & - & - & - & 1 & 0 & 2 & - & 1 & - & 4 & 4 & 465 \\
\hline Home range & 0 & - & 0 & - & - & 0 & 2 & - & - & 1 & 3 & 5 & 361 \\
\hline Island status & 1 & 1 & 0 & - & - & 0 & - & - & - & - & 2 & 4 & 0 \\
\hline
\end{tabular}




\begin{tabular}{lrlllllllllllr}
\hline Activity period & 1 & - & 0 & - & - & 0 & 1 & - & - & - & 2 & 4 & 455 \\
Migratory behavior & - & - & - & - & - & - & - & - & 0 & - & 0 & 1 & 0 \\
\hline Reproductive & & & & & & & & & & & & & \\
\hline Gestation length & $\mathbf{2}$ & $\mathbf{0}$ & $\mathbf{2}$ & $\mathbf{1}$ & $\mathbf{1}$ & $\mathbf{1}$ & - & $\mathbf{2}$ & - & $\mathbf{1}$ & $\mathbf{1 0}$ & $\mathbf{8}$ & $\mathbf{5 6 4}$ \\
Litters year & 0 & 1 & - & 1 & 1 & 1 & - & - & 2 & 1 & 7 & 7 & 309 \\
Weaning age & - & - & - & $\mathbf{2}$ & $\mathbf{1}$ & $\mathbf{2}$ & - & $\mathbf{1}$ & - & $\mathbf{1}$ & $\mathbf{7}$ & $\mathbf{5}$ & $\mathbf{5 2 7}$ \\
Litter size & $\mathbf{1}$ & $\mathbf{0}$ & $\mathbf{0}$ & - & $\mathbf{1}$ & $\mathbf{1}$ & - & - & $\mathbf{0}$ & $\mathbf{2}$ & $\mathbf{5}$ & $\mathbf{7}$ & $\mathbf{5 8 7}$ \\
Age sexual maturity & 1 & 0 & 0 & - & 1 & 0 & - & - & - & 2 & 4 & 6 & 530 \\
Reproductive rate & - & - & - & - & - & - & 2 & - & 0 & - & 2 & 2 & 0 \\
Interbirth interval & 0 & 0 & 0 & - & - & 0 & - & - & - & 1 & 1 & 5 & 408 \\
Age at first breeding & - & - & - & - & - & 0 & - & - & - & - & 0 & 1 & 281 \\
Age at eye opening & - & - & - & - & - & 0 & - & - & - & - & 0 & 1 & 247 \\
\hline Total evaluated traits & 12 & 7 & 10 & 7 & $25 \dagger$ & 17 & 11 & 5 & 11 & 14 & - & - & - \\
\hline Number of species & 355 & 867 & 229 & 4030 & 1513 & 4030 & 4420 & 5020 & 125 & 2761 & - & - & - \\
\hline
\end{tabular}

*This study selected these variables based on previous global comparative studies that indicated these are the most relevant traits for mammals.

$\dagger$ The entire list of tested variables for this study was not provided, the text indicates a database with 25 traits was gathered.

\section{References}

1. Purvis, A., et al., Predicting extinction risk in declining species. Proceedings of the Royal Society of London B Biological Sciences, 2000. 267(1456): p. 1947-1952.

2. Jones, K.E., A. Purvis, and J.L. Gittleman, Biological correlates of extinction risk in bats. American Naturalist, 2003. 161(4): p. 601-614.

3. Cardillo, M., et al., Human population density and extinction risk in the world's carnivores. PLoS Biology, 2004. 2(7): p. 909-914.

4. $\quad$ Cardillo, M., et al., Multiple causes of high extinction risk in large mammal species. Science, 2005. 309: p. 12391241.

5. Cardillo, M., et al., Latent extinction risk and the future battlegrounds of mammal conservation. Proceedings of the National Academy of Sciences of the United States of America, 2006. 103(11): p. 4157-4161.

6. Cardillo, M., et al., The predictability of extinction: biological and external correlates of decline in mammals. Proceedings of the Royal Society B: Biological Sciences, 2008. 275: p. 1441-1448.

7. Davidson, A.D., et al., Multiple ecological pathways to extinction in mammals. Proceedings of the National Academy of Sciences of the United States of America, 2009. 106(26): p. 10702-10705.

8. Fritz, S.A., O.R.P. Bininda-Emonds, and A. Purvis, Geographical variation in predictors of mammalian extinction risk: big is bad, but only in the tropics. Ecology Letters, 2009. 12(6): p. 538-549.

9. Davidson, A.D., et al., Drivers and hotspots of extinction risk in marine mammals. Proceedings of the National Academy of Sciences of the United States of America, 2012. 109(9): p. 3395-3400.

10. González-Suárez, M. and E. Revilla, Variability in life-history and ecological traits is a buffer against extinction in mammals. Ecology Letters, 2013. 16(2): p. 242-251. 


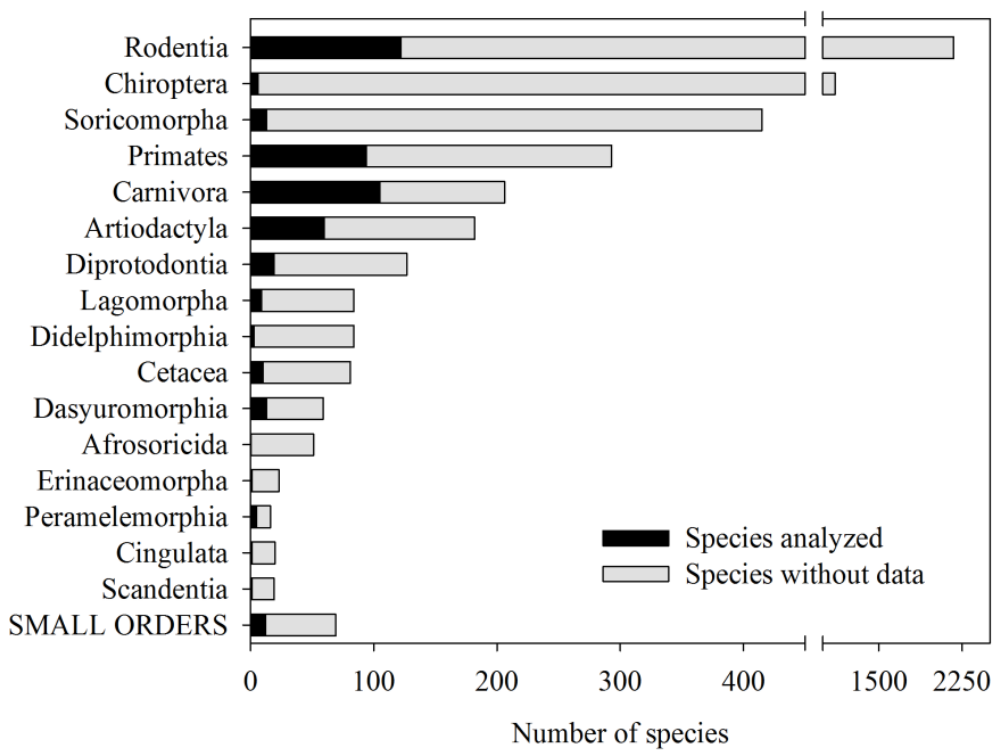

Figure S1. Number of species from each mammalian order for which data was available and hence included in our analyses (in black) in comparison with the known extant diversity of the order (in grey). SMALL ORDERS aggregates data for orders with <20 extant species: Dermoptera, Hyracoidea, Macroscelidea, Microbiotheria, Monotremata, Notoryctemorphia, Paucituberculata, Perissodactyla, Pholidota, Pilosa, Proboscidea, Sirenia, Tubulidentata.

\section{Body mass allometry}

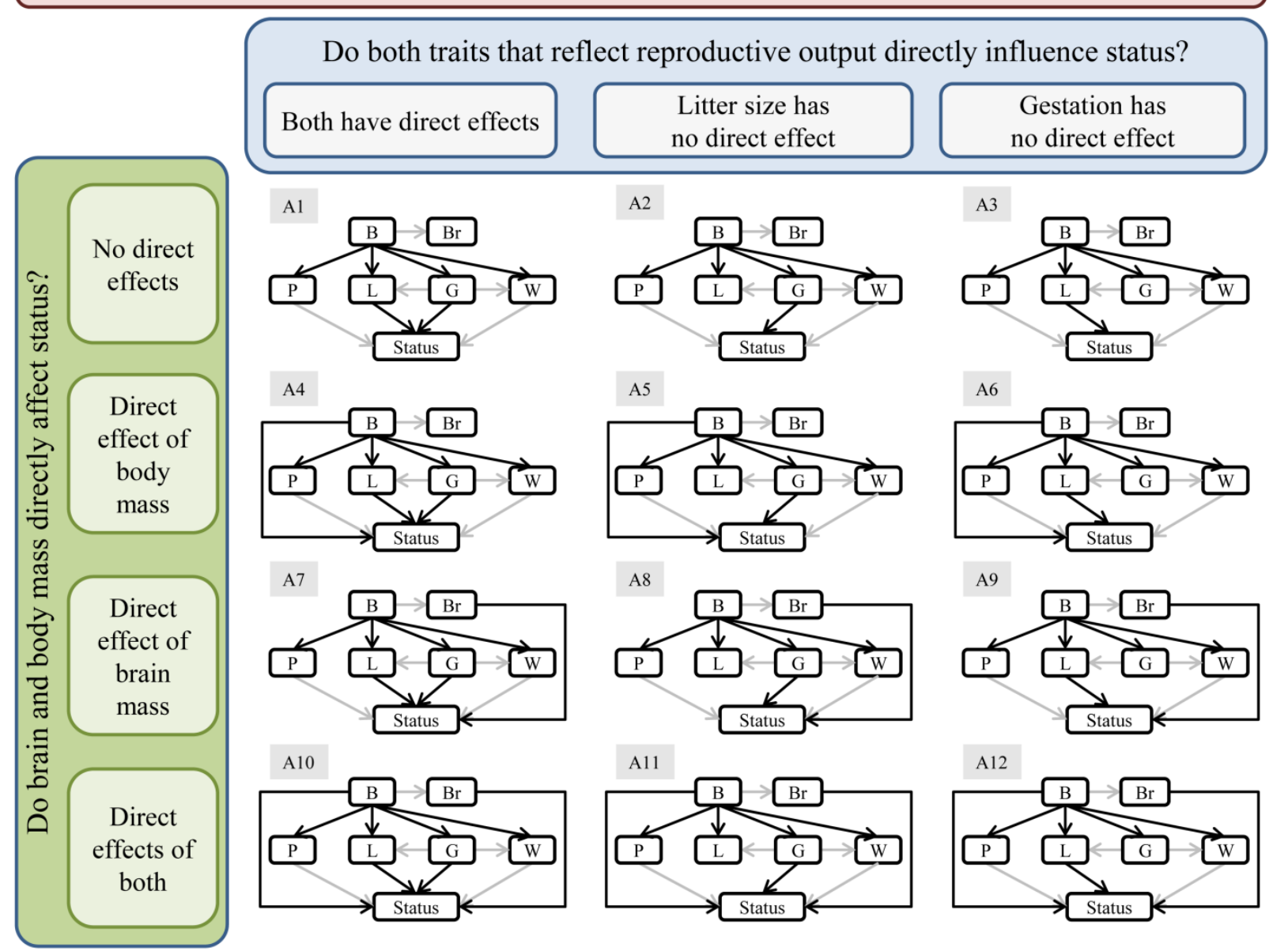


Figure S2. Path models tested under the Body mass allometry scenario which reflects the current paradigm in macroecological comparative studies of extinction risk which focus more on the role of body size, rarely considering brain size. Body mass (B), brain mass (Br), litter size $(\mathrm{L})$, gestation length $(\mathrm{G})$, weaning age $(\mathrm{W})$, population density $(\mathrm{P})$, and vulnerability to extinction based on the IUCN Red List categories (Status). Grey arrows indicate known relationships included in all models. 


\section{Brain costs and benefits}

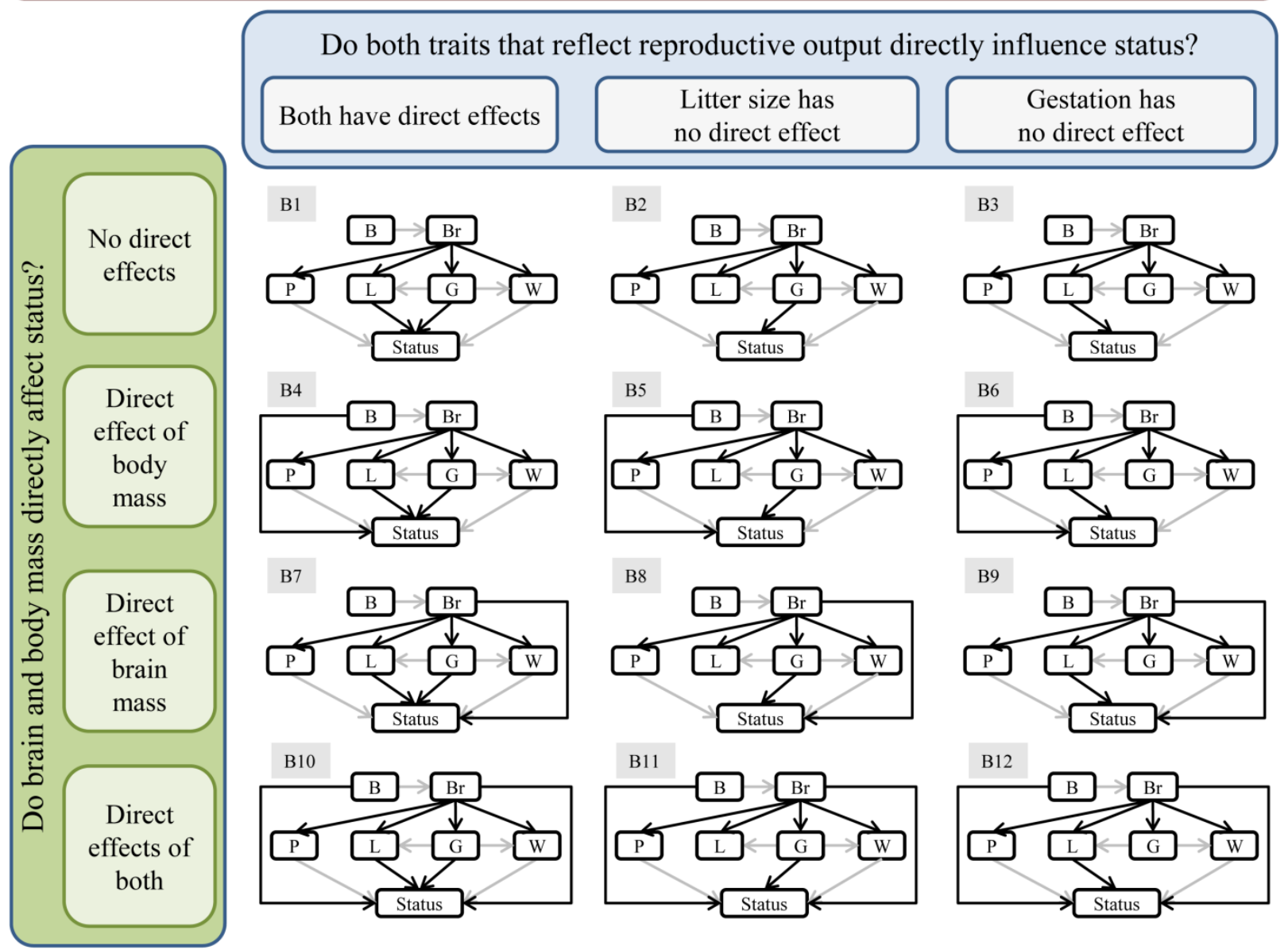

Figure S3. Path models tested under the Brain costs and benefits scenario which emphasizes the role of brain size, proposing that previously found correlations between body size and life-history and ecology are best explained by brain size. Body mass (B), brain mass (Br), litter size $(\mathrm{L})$, gestation length $(\mathrm{G})$, weaning age $(\mathrm{W})$, population density $(\mathrm{P})$, and vulnerability to extinction based on the IUCN Red List categories (Status). Grey arrows indicate known relationships included in all models. 


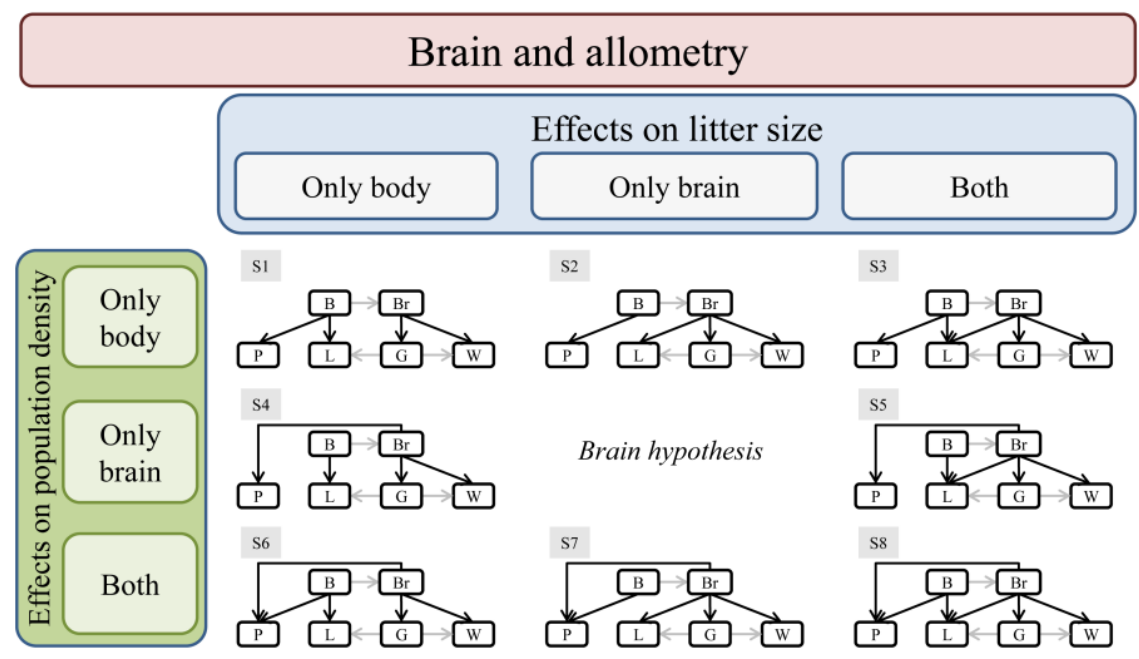

Figure S4. Step 1 of the definition of the path models tested under the Brain and allometry scenario. This scenario proposes that both brain and body mass influence life-history and ecological traits. Step 1 was designed to compare different evolutionary relationships between brain and body mass with life-history and population density traits. Body mass (B), brain mass $(\mathrm{Br})$, litter size $(\mathrm{L})$, gestation length $(\mathrm{G})$, weaning age $(\mathrm{W})$, and population density $(\mathrm{P})$. Grey arrows indicate known relationships included in all models.

A) Conceptual framework

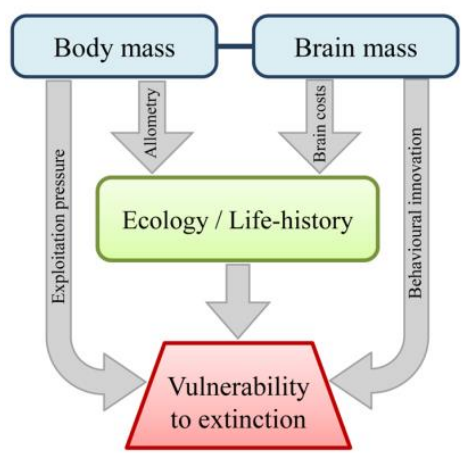

B) Path analyses results

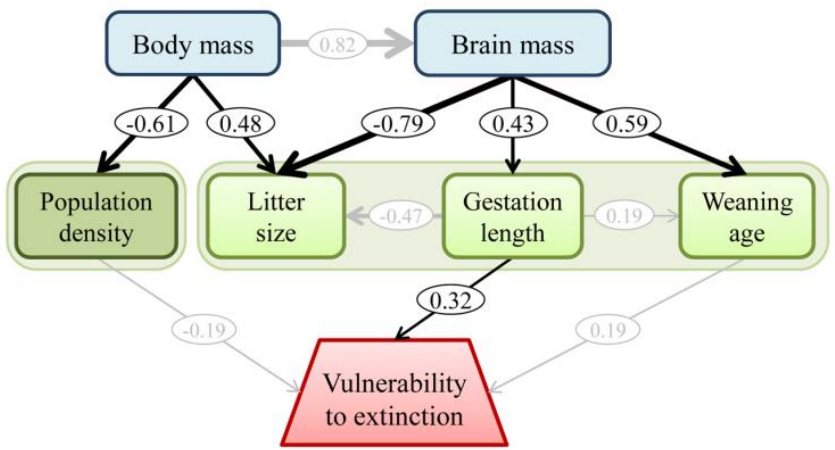

Figure S5. Path diagram results showing the empirical relationships among body mass, brain mass, life history, ecology, and vulnerability to extinction as described by the models best supported by the data (Table S2). These results are based on a dataset that excluded all mammals listed as threatened by the IUCN based on criteria C and/or D (which indicate small population size). The full dataset results are presented in figure 1 of the main text. The width of the arrows indicates their relative importance, and the numbers represent the averaged standardized slope coefficients. Solid arrows represent relationships supported in the model best supported by the data. Grey arrows indicate known relationships included in all models. 


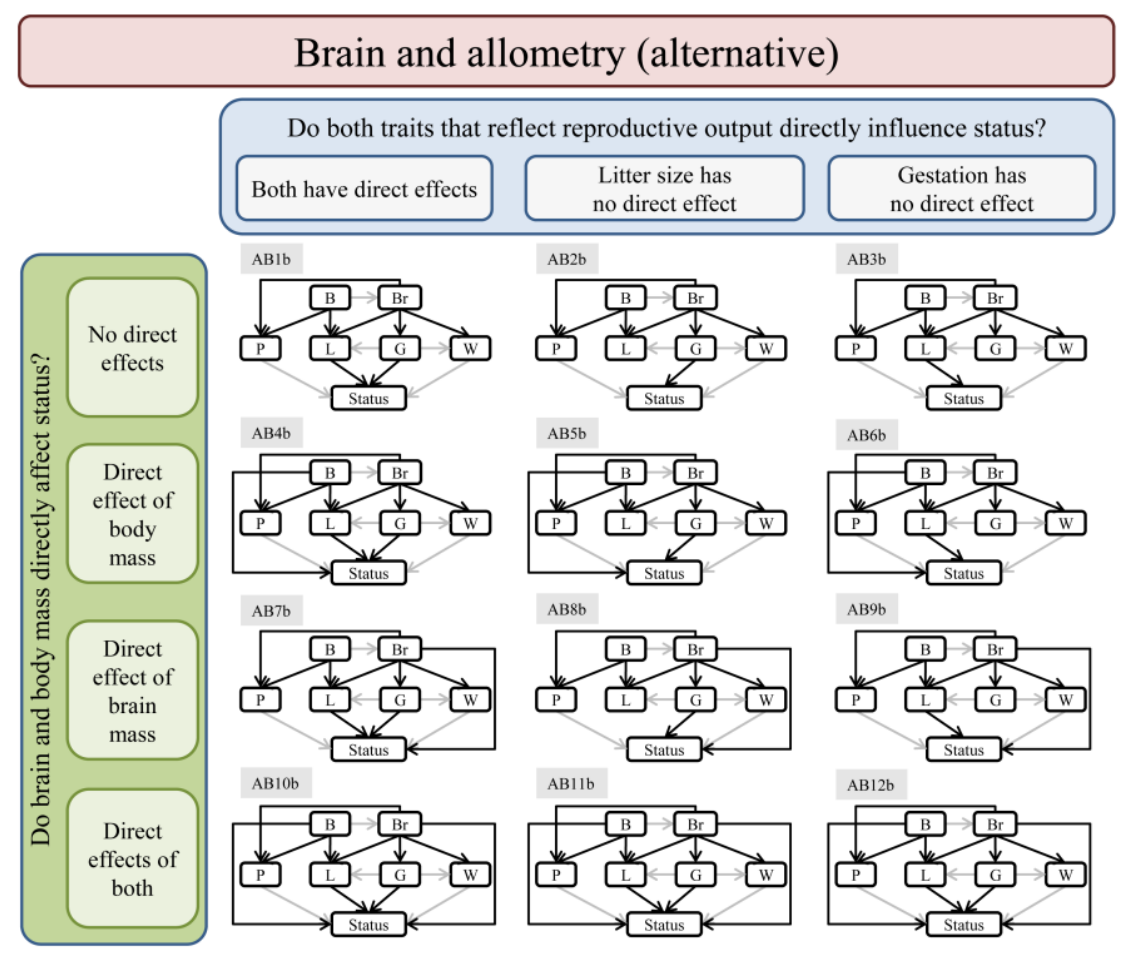

Figure S6. Path models tested under the Brain and allometry scenario based on the best supported model from step 1 (Model S8, Fig. S4). This scenario proposes that a combination of allometric effects and energetic costs of brain mass influence life-history and ecological traits. Body mass (B), brain mass (Br), litter size (L), gestation length (G), weaning age (W), population density (P), and vulnerability to extinction based on the IUCN Red List categories (Status). Grey arrows indicate known relationships included in all models. 
Table S2. Ranking of all path models tested based on CICc values. Model codes correspond to diagrams presented in figures 2, S2-S3. For each model we report the CICc value, $\triangle \mathrm{CICc}$ value, CICc weights $(\omega), \mathrm{C}$-statistic (C), and $P$-values of the C-statistic, where significant $P$-values indicate the model is rejected by the data. Models were based on 474 mammalian species (Fig. S1). Supported models $(\triangle \mathrm{CICc}<2)$ are highlighted in bold.

\begin{tabular}{|c|c|c|c|c|c|c|}
\hline Scenario & Model & CICc & $\Delta \mathrm{CICc}$ & $\omega$ & $\mathbf{C}$ & $P$-value \\
\hline Brain and allometry & AB2 & $\mathbf{5 7 . 8 7}$ & 0.00 & 0.31 & 20.37 & 0.44 \\
\hline Brain and allometry & AB5 & 59.29 & 1.42 & 0.15 & 19.62 & 0.35 \\
\hline Brain and allometry & AB8 & 59.56 & 1.69 & 0.13 & 19.89 & 0.34 \\
\hline Brain and allometry & AB1 & 59.73 & 1.86 & 0.12 & 20.06 & 0.33 \\
\hline Brain and allometry & $\mathrm{AB} 11$ & 60.76 & 2.89 & 0.07 & 18.90 & 0.27 \\
\hline Brain and allometry & AB4 & 61.15 & 3.28 & 0.06 & 19.30 & 0.25 \\
\hline Brain and allometry & $\mathrm{AB} 7$ & 61.43 & 3.55 & 0.05 & 19.57 & 0.24 \\
\hline Brain and allometry & AB3 & 62.56 & 4.69 & 0.03 & 25.06 & 0.20 \\
\hline Brain and allometry & AB10 & 62.69 & 4.82 & 0.03 & 18.65 & 0.18 \\
\hline Brain and allometry & AB9 & 64.49 & 6.62 & 0.01 & 24.82 & 0.13 \\
\hline Brain and allometry & AB6 & 64.62 & 6.75 & 0.01 & 24.95 & 0.13 \\
\hline Brain and allometry & $\mathrm{AB} 12$ & 65.46 & 7.58 & 0.01 & 23.60 & 0.10 \\
\hline Brain & $\mathrm{B} 2$ & 73.19 & 15.31 & 0.00 & 37.84 & 0.02 \\
\hline Brain & B5 & 74.48 & 16.61 & 0.00 & 36.98 & 0.01 \\
\hline Brain & B8 & 74.59 & 16.72 & 0.00 & 37.09 & 0.01 \\
\hline Brain & B1 & 74.92 & 17.05 & 0.00 & 37.42 & 0.01 \\
\hline Brain & B11 & 75.94 & 18.07 & 0.00 & 36.27 & 0.01 \\
\hline Brain & B4 & 76.33 & 18.46 & 0.00 & 36.66 & 0.01 \\
\hline Brain & B7 & 76.61 & 18.74 & 0.00 & 36.93 & 0.01 \\
\hline Brain & B3 & 77.76 & 19.89 & 0.00 & 42.42 & 0.01 \\
\hline Brain & B10 & 77.86 & 19.99 & 0.00 & 36.01 & $<0.01$ \\
\hline Brain & B9 & 79.68 & 21.81 & 0.00 & 42.18 & $<0.01$ \\
\hline Brain & B6 & 79.81 & 21.94 & 0.00 & 42.31 & $<0.01$ \\
\hline Brain & B12 & 80.64 & 22.77 & 0.00 & 40.96 & $<0.01$ \\
\hline Allometry & $\mathrm{A} 2$ & 115.03 & 57.16 & 0.00 & 79.69 & $<0.01$ \\
\hline Allometry & A5 & 116.44 & 58.57 & 0.00 & 78.94 & $<0.01$ \\
\hline Allometry & A8 & 116.62 & 58.75 & 0.00 & 79.12 & $<0.01$ \\
\hline Allometry & $\mathrm{A} 1$ & 116.79 & 58.92 & 0.00 & 79.29 & $<0.01$ \\
\hline Allometry & A11 & 117.81 & 59.94 & 0.00 & 78.13 & $<0.01$ \\
\hline Allometry & A4 & 118.20 & 60.33 & 0.00 & 78.53 & $<0.01$ \\
\hline Allometry & A7 & 118.48 & 60.60 & 0.00 & 78.80 & $<0.01$ \\
\hline Allometry & A3 & 119.54 & 61.67 & 0.00 & 84.20 & $<0.01$ \\
\hline Allometry & A10 & 119.73 & 61.86 & 0.00 & 77.88 & $<0.01$ \\
\hline Allometry & A9 & 121.51 & 63.64 & 0.00 & 84.01 & $<0.01$ \\
\hline Allometry & A6 & 121.63 & 63.76 & 0.00 & 84.13 & $<0.01$ \\
\hline Allometry & A12 & 122.51 & 64.63 & 0.00 & 82.83 & $<0.01$ \\
\hline
\end{tabular}


Table S3. Ranking of all path models tested based on CICc values excluding species listed as threatened under criteria $\mathrm{C}$ and $\mathrm{D}$ by the IUCN $(\mathbf{N = 4 5 3 )}$ ). Model codes correspond to diagrams presented in figures $2, \mathrm{~S} 2-\mathrm{S} 3$. For each model we report the CICc value, $\Delta \mathrm{CICc}$ value, CICc weights $(\omega), \mathrm{C}$-statistic (C), and $P$-values of the $\mathrm{C}$-statistic, where significant $P$-values indicate the model is rejected by the data. Supported models $(\Delta \mathrm{CICc}<2)$ are highlighted in bold.

\begin{tabular}{|c|c|c|c|c|c|c|}
\hline Scenario & Model & CICc & $\Delta \mathrm{CICc}$ & $\omega$ & $\mathbf{C}$ & $P$-value \\
\hline Brain and allometry & AB2 & 53.46 & 0.00 & 0.32 & 15.88 & 0.72 \\
\hline Brain and allometry & AB5 & 54.67 & 1.22 & 0.18 & 14.91 & 0.67 \\
\hline Brain and allometry & AB8 & 55.04 & 1.59 & 0.15 & 15.28 & 0.64 \\
\hline Brain and allometry & $\mathrm{AB} 1$ & 55.51 & 2.05 & 0.12 & 15.75 & 0.61 \\
\hline Brain and allometry & $\mathrm{AB} 11$ & 56.45 & 3.00 & 0.07 & 14.50 & 0.56 \\
\hline Brain and allometry & AB4 & 56.73 & 3.27 & 0.06 & 14.78 & 0.54 \\
\hline Brain and allometry & $\mathrm{AB} 7$ & 57.10 & 3.64 & 0.05 & 15.15 & 0.51 \\
\hline Brain and allometry & $\mathrm{AB} 10$ & 58.55 & 5.09 & 0.03 & 14.40 & 0.42 \\
\hline Brain and allometry & AB6 & 61.08 & 7.62 & 0.01 & 21.32 & 0.26 \\
\hline Brain and allometry & $\mathrm{AB} 9$ & 61.38 & 7.92 & 0.01 & 21.62 & 0.25 \\
\hline Brain and allometry & $\mathrm{AB} 3$ & 62.42 & 8.97 & 0.00 & 24.84 & 0.21 \\
\hline Brain and allometry & $\mathrm{AB} 12$ & 62.96 & 9.51 & 0.00 & 21.01 & 0.18 \\
\hline Brain & $\mathrm{B} 2$ & 66.78 & 13.33 & 0.00 & 31.37 & 0.09 \\
\hline Brain & B5 & 67.90 & 14.44 & 0.00 & 30.32 & 0.06 \\
\hline Brain & B8 & 67.99 & 14.53 & 0.00 & 30.41 & 0.06 \\
\hline Brain & B1 & 68.73 & 15.28 & 0.00 & 31.15 & 0.05 \\
\hline Brain & B11 & 69.67 & 16.21 & 0.00 & 29.91 & 0.04 \\
\hline Brain & B4 & 69.94 & 16.49 & 0.00 & 30.18 & 0.04 \\
\hline Brain & B7 & 70.31 & 16.86 & 0.00 & 30.55 & 0.03 \\
\hline Brain & B10 & 71.75 & 18.29 & 0.00 & 29.80 & 0.02 \\
\hline Brain & B6 & 74.30 & 20.85 & 0.00 & 36.73 & 0.01 \\
\hline Brain & B9 & 74.60 & 21.14 & 0.00 & 37.02 & 0.01 \\
\hline Brain & B3 & 75.66 & 22.20 & 0.00 & 40.25 & 0.01 \\
\hline Brain & B12 & 76.18 & 22.72 & 0.00 & 36.42 & 0.01 \\
\hline Allometry & $\mathrm{A} 2$ & 110.55 & 57.10 & 0.00 & 75.14 & $<0.01$ \\
\hline Allometry & A5 & 111.76 & 58.30 & 0.00 & 74.18 & $<0.01$ \\
\hline Allometry & A8 & 112.06 & 58.61 & 0.00 & 74.48 & $<0.01$ \\
\hline Allometry & $\mathrm{A} 1$ & 112.53 & 59.08 & 0.00 & 74.95 & $<0.01$ \\
\hline Allometry & A11 & 113.46 & 60.01 & 0.00 & 73.70 & $<0.01$ \\
\hline Allometry & A4 & 113.74 & 60.28 & 0.00 & 73.98 & $<0.01$ \\
\hline Allometry & A7 & 114.11 & 60.65 & 0.00 & 74.35 & $<0.01$ \\
\hline Allometry & A10 & 115.55 & 62.09 & 0.00 & 73.60 & $<0.01$ \\
\hline Allometry & A6 & 118.07 & 64.62 & 0.00 & 80.49 & $<0.01$ \\
\hline Allometry & A9 & 118.43 & 64.98 & 0.00 & 80.85 & $<0.01$ \\
\hline Allometry & $\mathrm{A} 3$ & 119.47 & 66.01 & 0.00 & 84.06 & $<0.01$ \\
\hline Allometry & A12 & 119.97 & 66.52 & 0.00 & 80.21 & $<0.01$ \\
\hline
\end{tabular}


Table S4. Ranking based on CICc values of the path models exploring evolutionary relationships in step 1 of the Brain and allometry scenario analysis.

Models S8 and S3 were selected as best supported by the data, because S8 is a version of S3 with an additional path we selected the simplest model (S3) as the basis to construct models in step 2 (Fig. 2 main text). We also explored an alternative set of models for step 2 using S8 (Fig. S6). Results were qualitatively the same (Table S5). Model codes correspond to diagrams presented in figure S4 (step 1). For each model we report the CICc value, $\triangle \mathrm{CICc}$ value, CICc weights ( $\omega), \mathrm{C}$-statistic (C), and $P$-values of the $\mathrm{C}$-statistic, where significant $P$-values indicate the model is rejected by the data. Supported models $(\Delta \mathrm{CICc}<2)$ are highlighted in bold.

\begin{tabular}{crrrcr}
\hline Models & CICc & $\Delta$ CICc & $\boldsymbol{\omega}$ & C & $\boldsymbol{P}$-value \\
\hline S8 & $\mathbf{4 5 . 8 7}$ & $\mathbf{0 . 0 0}$ & $\mathbf{0 . 6 7}$ & $\mathbf{1 4 . 8 2}$ & $\mathbf{0 . 2 5}$ \\
S3 & $\mathbf{4 7 . 5 6}$ & $\mathbf{1 . 7 0}$ & $\mathbf{0 . 2 9}$ & $\mathbf{1 8 . 6 5}$ & $\mathbf{0 . 1 8}$ \\
S5 & 51.62 & 5.76 & 0.04 & 22.71 & 0.07 \\
S7 & 56.36 & 10.50 & 0.00 & 27.45 & 0.02 \\
S2 & 58.07 & 12.20 & 0.00 & 31.28 & 0.01 \\
S6 & 63.54 & 17.68 & 0.00 & 34.63 & $<0.01$ \\
S1 & 65.25 & 19.38 & 0.00 & 38.46 & $<0.01$ \\
S4 & 69.31 & 23.44 & 0.00 & 42.52 & $<0.01$ \\
\hline
\end{tabular}

Table S5. Complete models for the Brain and allometry scenario based on the alternative best model from step 1 (Table S4). Model codes correspond to path models presented figure S6. For each model we report the CICc value, $\Delta$ CICc value, CICc weights $(\omega), \mathrm{C}$-statistic (C), and $P$-values of the $\mathrm{C}$-statistic, where significant $P$-values indicate the model is rejected by the data. Supported models $(\Delta \mathrm{CICc}<2)$ are highlighted in bold.

\begin{tabular}{lrrrrr}
\hline Models & CICc & $\Delta$ CICc & $\boldsymbol{\omega}$ & C & $\boldsymbol{P}$-value \\
\hline AB2b & $\mathbf{5 6 . 2 1}$ & $\mathbf{0 . 0 0}$ & $\mathbf{0 . 3 1}$ & $\mathbf{1 6 . 5 4}$ & $\mathbf{0 . 5 5}$ \\
AB5b & $\mathbf{5 7 . 6 4}$ & $\mathbf{1 . 4 3}$ & $\mathbf{0 . 1 5}$ & $\mathbf{1 5 . 7 9}$ & $\mathbf{0 . 4 7}$ \\
AB8b & $\mathbf{5 7 . 9 1}$ & $\mathbf{1 . 7 0}$ & $\mathbf{0 . 1 3}$ & $\mathbf{1 6 . 0 6}$ & $\mathbf{0 . 4 5}$ \\
AB1b & $\mathbf{5 8 . 0 8}$ & $\mathbf{1 . 8 7}$ & $\mathbf{0 . 1 2}$ & $\mathbf{1 6 . 2 3}$ & $\mathbf{0 . 4 4}$ \\
AB11b & 59.12 & 2.91 & 0.07 & 15.08 & 0.37 \\
AB4b & 59.51 & 3.30 & 0.06 & 15.47 & 0.35 \\
AB7b & 59.79 & 3.57 & 0.05 & 15.74 & 0.33 \\
AB3b & 60.90 & 4.69 & 0.03 & 21.23 & 0.38 \\
AB10b & 61.06 & 4.85 & 0.03 & 14.82 & 0.10 \\
AB9b & 62.84 & 6.63 & 0.01 & 20.99 & 0.18 \\
AB6b & 62.97 & 6.76 & 0.01 & 21.12 & 0.17 \\
AB12b & 63.82 & 7.60 & 0.01 & 19.77 & 0.14 \\
\hline
\end{tabular}




\section{Supplementary Results: Taxon-specific analyses}

There were minor differences within each order for the relationships between brain, body sizes and life history traits or among life history traits. We therefore had to modify slightly the causal links between traits to ensure that all conditional independencies were met in all models (Fig. S7).

As illustrated in figure S7 the differences in the tested models for the different orders are as follows. For Carnivora and Primates population density is not independent of brain size even when controlling for body size, it is however independent of body size when controlling for brain size. Thus, the causal link was modified to go from brain size to population density. For Primates, weaning age was not independent of litter size, thus a causal link was added from litter size to weaning age. The causal link between litter size and weaning age could be due to the fact that litter size is a proxy for neonate size, which influences weaning age. Alternatively, it may also be a result of the limited variability in litter in Primates. Finally, for Artiodactyla, weaning age depends of body size rather than brain size. Thus, the causal link was modified to go from body size to weaning age.

Note that because of the differences in causal links for the different orders, the models are not directly comparable, because the causal links involved are different.

\section{Taxon-specific models}

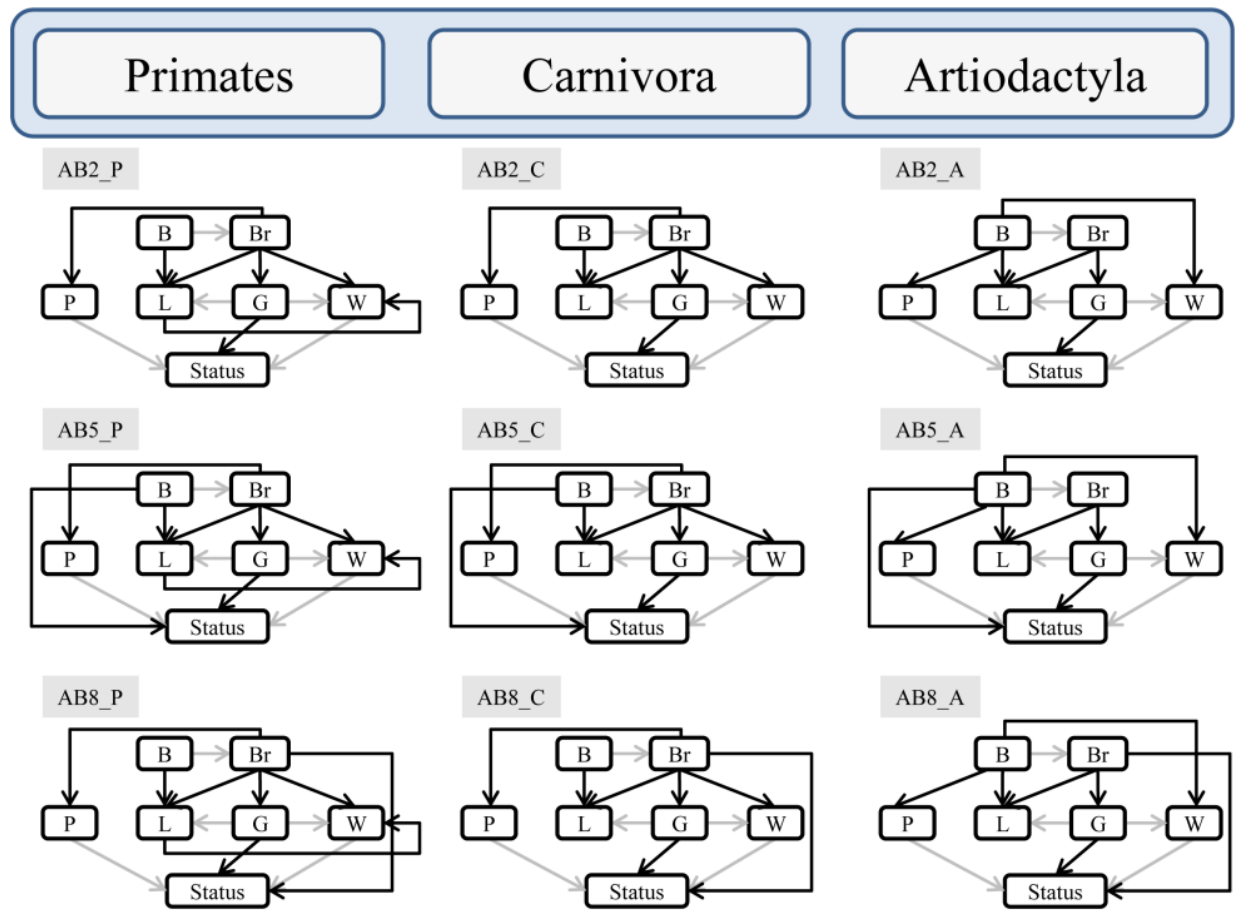

Figure S7. Path models for the three orders for which taxon-specific analyses were possible. Tested model included slight modifications of the three models supported in the overall analyses (AB2, AB5 and AB8, fig 2 main text). Body mass (B), brain mass $(\mathrm{Br})$, litter size $(\mathrm{L})$, gestation length $(\mathrm{G})$, weaning age $(\mathrm{W})$, population density $(\mathrm{P})$, and vulnerability to extinction based on the IUCN Red List categories (Status). Grey arrows indicate known relationships included in all models. 
Table S6. Ranking of the three best-supported models based on CICc values for the three orders for which taxonspecific analyses were possible. The table shows the model number only for comparison with the results of the complete database, although as mentioned above the models vary slightly between orders and when compared to those of the complete dataset. For each model we report the CICc value, and C-statistic (C). All C statistic values were non-significant, indicating that minimum set of conditional independencies were fulfilled by the observational data.

\begin{tabular}{crrr}
\hline Model & CICc & \multicolumn{1}{c}{ C } & $P$-value \\
\hline Primates & & & \\
\hline AB2_P & 63.02 & 16.08 & 0.59 \\
AB5_P & 60.12 & 10.12 & 0.86 \\
AB8_P & 58.58 & 8.58 & 0.93 \\
\hline Carnivora & & & \\
\hline AB2_C & 68.40 & 24.44 & 0.22 \\
AB5_C & 69.62 & 22.68 & 0.20 \\
AB8_C & 68.34 & 21.40 & 0.26 \\
\hline Artiodactyla & & & \\
\hline AB2_A & 65.82 & 13.14 & 0.87 \\
AB5_A & 69.18 & 12.18 & 0.84 \\
AB8_A & 69.55 & 12.55 & 0.82 \\
\hline
\end{tabular}

